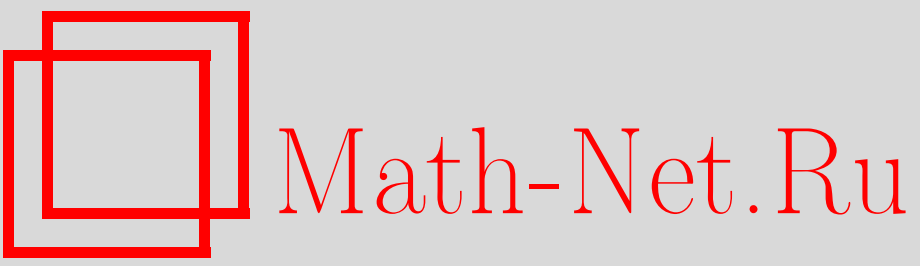

P. J. Szabłowski, $q$-Wiener and $(\alpha, q)$-Ornstein-Uhlenbeck processes. A generalization of known processes, Теория вероятн. и ее примен., 2011, том 56, выпуск 4, 742-772

DOI: https://doi.org/10.4213/tvp4421

Использование Общероссийского математического портала Math-Net.Ru подразумевает, что вы прочитали и согласны с пользовательским соглашением

http://www.mathnet.ru/rus/agreement

Параметры загрузки:

IP : 35.173 .219 .12

26 апреля 2023 г., 15:37:47 


\title{
$q$-WIENER AND $(\alpha, q)$-ORNSTEIN-UHLENBECK PROCESSES. A GENERALIZATION OF KNOWN PROCESSES
}

\begin{abstract}
Мы собираем разбросанные в литературе и доказываем некоторые новые свойства двух марковских процессов, во многом сходных с винеровским процессом и процессом Орнштейна-Уленбека. Хотя рассматриваемые в настоящей работе процессы были определены в контексте некоммутативной вероятности или через квадратические связки (harnesses), мы определяем их заново как своего рода обобщение «для непрерывного времени» простых симметричных процессов с дискретным временем, удовлетворяющих простым условиям на форму первых двух условных моментов. Конечномерные распределения первого из этих процессов (скажем, $\left.\mathbf{X}=\left(X_{t}\right)_{t \geqslant 0}\right)$, называемого $q$-винеровским, зависят от одного параметра $q \in(-1,1]$, а конечномерные распределения второго (скажем, $\left.\mathbf{Y}=\left(Y_{t}\right)_{t \in \mathbf{R}}\right)$, называемого $(\alpha, q)$-процессом Орнштейна-У ленбека, - от двух параметров $(\alpha, q) \in(0, \infty) \times(-1,1]$. Первый процесс имеет с винеровским то общее, что при $q=1$ он сам является винеровским, а при $|q|<1$ для любого $n \geqslant 1$ процесс $t^{n / 2} H_{n}\left(X_{t} / \sqrt{t} \mid q\right)$, где $\left(H_{n}\right)_{n \geqslant 0}-$ так называемые $q$-полиномы Эрмита, является мартингалом. Он, однако, не имеет ни независимых приращений, ни модификации с непрерывными траекториями. Второй процесс сходен с процессом Орнштейна-Уленбека. При $q=1$ он превращается в классический процесс Орнштейна-Уленбека. При $|q|<1$ он является стационарным процессом с корреляционной функцией $\exp (-\alpha|t-s|)$ и обладает многими свойствами, сходными со свойствами классической версии. Эти процессы представляются нам захватывающим предметом для исследования, предлагающим много интересных открытых вопросов.
\end{abstract}

Ключевые слова и Орнштейна-Уленбека, полиномиальное мартингальное свойство, $q$-гауссовские распределения, квадратические связки (harnesses), строго марковское свойство, феллеровская непрерывность, $q$-полиномы Эрмита, полиномы Аль-Салама-Чихары.

1. Introduction. As announced in the abstract, we are going to define two time-continuous families of Markov processes. One of them will

*Department of Mathematics and Information Sciences, Warsaw University of Technology, pl. Politechniki 1, 00-661 Warszawa, Poland; e-mail: pszablowski@elka.pw.edu.pl, pawel.szablowski@gmail.com 
resemble Wiener process and the other Ornstein-Uhlenbeck (OU) process. They will be indexed (apart from the time parameter) by an additional parameter $q \in(-1,1]$. In the case $q=1$ these processes are the classical Wiener and the OU processes. Of course for the OU process there will be an additional parameter $\alpha>0$ responsible for the covariance function of the process. For $q \in(-1,1)$ both processes will assume their values in a compact space: the $(\alpha, q)$-OU process in $[-2 / \sqrt{1-q}, 2 / \sqrt{1-q}]$, while for the $q$-Wiener process $\left(X_{t}\right)_{t \geqslant 0}$ we will have: $X_{t} \in[-2 \sqrt{t /(1-q)}, 2 \sqrt{t /(1-q)}]$. The one-dimensional probabilities and the transitional probabilities of these processes will be given explicitly. Moreover two families of polynomials, orthogonal with respect to these measures, will also be presented. Some properties of the conditional expectations given the past and also the past and the future will be exposed. Martingale properties, as well as some properties of the sample path of these processes will be described. Thus quite detailed knowledge concerning these processes will be presented.

The processes that we are going to reintroduce have appeared already in 1997 in an excellent paper [20] as an offspring and a particular case of some noncommutative probability model. Some of the properties of these processes particularly those associated with their martingale behavior of some functions of these processes were also discussed in this paper. Since 1997 there appeared couple of papers on the properties of $q$-Gaussian distributions. See, e.g., [11], [23], [21].

There is also a different path of research followed by Włodek Bryc, Wojtek Matysiak and Jacek Wesołowski, see, e.g., [9], [10], [13]. Their starting point is a continuous-time process that satisfies several (exactly five) conditions on the covariance function and on the first and the second conditional moments. Those are the so-called quadratic harnesses characterized by five parameters. Under resulting assumptions they proved that these processes are Markov and also stated several properties of the families of polynomials that orthogonalize the transitional and the one-dimensional probabilities. They gave also several examples illustrating the developed theory. One of the processes considered by them is the so-called $q$-Brownian process. Four of five possible parameters are equal to zero and the fifth one can be identified with the parameter $q$ considered in this paper. As far as the one-dimensional probabilities and the transitional probabilities are concerned this process is identical with the $q$-Wiener process introduced and analyzed in this paper. They did not however work on the properties of the $q$-Brownian process. It appeared as a by-product of their interest in quadratic harnesses. Bryc, Matysiak, and Wesolowski were mostly interested in the general problem of existence of quadratic harnesses. That is why $(\alpha, q)$-OU process has not appeared in their works.

What we are aiming to do is to reintroduce these processes via certain discrete time one-dimensional, time symmetric random process (1TSP) by 
the so to say «continuation of time» or maybe more precisely as the processes that sampled at certain discrete moments have the properties of the 1TSP. This discrete time process was defined in a purely classical probability context. Moreover it is very simple and intuitive. Its simplicity surprised Richard Askey «that it has no $q$ in the statement of the problem» as he puts it in the Forward to [5]. Thus these 1TSP can be simple models of some phenomena observed in the recently intensively developing $q$-series theory. 1TSP first appeared in 2001 in [1] and has been studied in detail recently, see, e.g., [2], [3], [7], [8].

Besides we derive these processes under fewer assumptions (than one would need while following the quadratic harnesses path) on the first and the second conditional moments (we need only 2). The construction is also different. As mentioned earlier our starting point is a discrete time 1TSP. The $q$-Wiener process is obtained as continuous time transformation of the process that we call the $(\alpha, q)$-OU process (like in the classical, i.e., $q=1$, case) which, on its side, is obtained as a continuous time generalization of the discrete time process (1TSP). Besides we list many properties of the $q$-Wiener process that justify its name (are sort of $q$-analogies of well-known martingale properties of the Wiener process). Some of these properties can be derived from the definition of the $q$-Brownian process presented in [9]. They are not however stated it explicitly.

That is why Section 2 will be dedicated to the definition of 1TSP's and recollection of their basic properties. Since our approach is totally commutative, $q$ by no means is an operator. It is a number parameter. Yet we are touching the $q$-series theory and the special functions.

We think that the processes presented in this paper are the fascinating objects to study. As mentioned before, for $|q|<1$, the $q$-Wiener process has many properties similar to the ordinary Brownian motion, but is not an independent increment process. Besides we present a few properties of trajectories of the processes discussed in this paper. Properties that, although can be relatively easily deduced, were never stated in the above mentioned papers, where these processes appeared for the first time.

The paper is organized as follows. As we mentioned, in Section 2 we recall the definition and summarize basic properties of the 1TSP's. Section 3 is still dedicated mainly to certain auxiliary properties of the discrete time 1TSP's that are necessary to perform our construction. In Section 4 we introduce the continuous-time version of $1 \mathrm{TSP}((\alpha, q)$-OU process $)$ and prove its existence. Then we define the $q$-Wiener as a continuous time transformation of the $(\alpha, q)$-OU process. Later parts of this section are devoted to presentation of the two processes and listing or proving some of their properties. We indicate their connection with the emerging quadratic harness theory.

We point out also here where is the mistake causing that Wesołowski's 
martingale characterization of the Wiener process contained in [19] is not true. The fact that it is not true was already known to Wesolowski (see, e.g., [9], where processes denying his characterization are pointed out).

Section 5 presents some obvious open problems that come to mind almost directly. The last Section 6 contains lengthy proofs of the results from the previous sections.

It is known that 1TSP exists with parameter $q>1$ (see [14]). Its transition distribution is then discrete. We show in this paper that the $q$ Wiener and the $(\alpha, q)$-Ornstein-Uhlenbeck processes do not exist for $q>1$.

2. One dimensional time symmetric random processes. By 1TSP's we mean square integrable random field $\mathbf{X}=\left\{X_{n}\right\}_{n \in \mathbf{Z}}$ indexed by the integers, with nonsingular all covariance matrices and constant first two moments, that satisfy the following two sets of conditions:

$$
\exists a, b \in \mathbf{R}, \forall n \in \mathbf{Z}: \quad \mathbf{E}\left(X_{n} \mid \mathscr{F}_{\neq n}\right)=a\left(X_{n-1}+X_{n+1}\right)+b \quad \text { a.s. }
$$

and

$$
\begin{aligned}
& \exists A, B, C, D \in \mathbf{R}, \forall n \in \mathbf{Z}: \quad \mathbf{E}\left(X_{n}^{2} \mid \mathscr{F}_{\neq n}\right) \\
& \quad=A\left(X_{n-1}^{2}+X_{n+1}^{2}\right)+B X_{n-1} X_{n+1}+D\left(X_{n-1}+X_{n+1}\right)+C \quad \text { a.s. }
\end{aligned}
$$

where $\mathscr{F}_{\neq m}:=\sigma\left(X_{k}: k \neq m\right)$.

Let us define also $\sigma$-algebras $\mathscr{F}_{\leqslant m}:=\sigma\left(X_{k}: k \leqslant m\right), \mathscr{F}_{\geqslant m}:=\sigma\left(X_{k}: k \geqslant\right.$ $m)$, and $\mathscr{F}_{\leqslant m, \geqslant j}:=\sigma\left(X_{k}: k \leqslant m \vee k \geqslant j\right)$.

Nonsingularity of covariance matrices implies that all random variables $X_{n}$ are non-degenerate and there is no loss of generality in assuming that $\mathbf{E} X_{k}=0$ and $\mathbf{E} X_{k}^{2}=1$ for all $k \in \mathbf{Z}$, which implies $b=0$.

It has been shown in [7] that (2.1) implies $L_{2}$-stationarity (stationarity in the wider sense) of $\mathbf{X}$. Since the case $\rho:=\operatorname{corr}\left(X_{0}, X_{1}\right)=0$ contains sequences of independent random variables (which satisfy (2.1) and (2.2) but can have arbitrary distributions), we shall exclude it from the considerations. Observe that non-singularity of the covariance matrices implies $|\rho|<1$. By Theorem 3.1 from [1] (see also [7, Theorems 1, 2]), we have $\operatorname{corr}\left(X_{0}, X_{k}\right)=$ $\rho^{|k|}$. Moreover the one-sided regressions are linear:

$$
\mathbf{E}\left(X_{m} \mid \mathscr{F}_{\leqslant 0}\right)=\rho^{m} X_{0}=\mathbf{E}\left(X_{-m} \mid \mathscr{F}_{\geqslant 0}\right), \quad m \geqslant 1 .
$$

It turns out that parameters $a, \rho, A, B, C$ are related to one another. In [1] and [8] it was shown that $D=0$ and one can redefine parameters by introducing new parameter

$$
q=\frac{\rho^{4}+B(\rho+1 / \rho)^{2}-1}{1+\rho^{4}\left(B(\rho+1 / \rho)^{2}-1\right)}
$$


and express parameters $A, B, C$ with the help of $\rho$ and $q$ only in the following manner:

$$
A=\frac{\rho^{2}\left(1-q \rho^{2}\right)}{\left(\rho^{2}+1\right)\left(1-q \rho^{4}\right)}, \quad B=\frac{\rho^{2}\left(1-\rho^{2}\right)(1+q)}{\left(\rho^{2}+1\right)\left(1-q \rho^{4}\right)}, \quad C=\frac{\left(1-\rho^{2}\right)^{2}}{1-q \rho^{4}} .
$$

We can rephrase and summarize the results of [1] and [7] in the following way. Each 1TSP is characterized by two parameters $\rho$ and $q$. For $q$ outside the set $[-1,1] \cup\left\{1 / \rho^{2 / n}: n \in \mathbf{N}\right\}$ the 1TSP's do not exist. For $0<|\rho|<1$ and $q \in[-1,1]$ the 1TSP's exist and all their finite dimensional distributions are uniquely determined and known. Also it follows from [1] and [2] that the 1TSP's are Markov processes. The case $0<|\rho|<1$ and $q \in\left\{1 / \rho^{2 / n}: n \in \mathbf{N}\right\}$ is treated in [14].

1TSP with $0<|\rho|<1$ and $q \in(-1,1]$ will be called regular.

We adopt notation traditionally used in $\ll q$-series theory»: $(a ; q)_{0}=1$,

$$
\begin{gathered}
(a ; q)_{n}=\prod_{i=0}^{n-1}\left(1-a q^{i}\right), \quad\left(a_{1}, \ldots, a_{k} ; q\right)_{n}=\prod_{i=1}^{k}\left(a_{i} ; q\right)_{n}, \\
{[0]_{q}=0, \quad[n]_{q}=1+\cdots+q^{n-1}, \quad n \geqslant 1,} \\
{[0]_{q} !=1, \quad[n]_{q} !=\prod_{i=1}^{n}[i]_{q},} \\
{\left[\begin{array}{l}
n \\
k
\end{array}\right]= \begin{cases}\frac{[n]_{q} !}{[k]_{q} ![n-k]_{q}} & \text { when } 0 \leqslant k \leqslant n, \\
0 & \text { when } k>n .\end{cases} }
\end{gathered}
$$

Notice that we have:

$$
(q ; q)_{n}=(1-q)^{n}[n]_{q} !, \quad\left[\begin{array}{l}
n \\
k
\end{array}\right]_{q}=\frac{(q ; q)_{n}}{(q ; q)_{k}(q ; q)_{n-k}} .
$$

As it is customary in $q$-series theory we will often abbreviate $(a ; q)_{n}$ and $\left(a_{1}, \ldots, a_{k} ; q\right)_{n}$ to $(a)_{n}$ and $\left(a_{1}, \ldots, a_{k}\right)_{n}$ if it will not cause misunderstanding.

To complete recollection of basic properties of the 1TSP let us introduce the so-called $q$-Hermite polynomials $\left\{H_{n}(x \mid q)\right\}_{n \geqslant-1}$ defined by the following recurrence:

$$
\forall n \geqslant 0: x H_{n}(x \mid q)=H_{n+1}(x \mid q)+[n]_{q} H_{n-1}(x \mid q),
$$

with $H_{-1}(x \mid q)=0, H_{0}(x \mid q)=1$.

$\mathrm{R}$ e $\mathrm{m}$ a $\mathrm{rk}$ 1. Comparing initial values and 3-term recurrences (see, e.g., [6]) one can easily notice that $\left\{H_{n}(x \mid 1)\right\}_{n \geqslant-1}$ are the so-called probabilistic Hermite polynomials (i.e., orthogonal with respect to the measure with density $\left.\exp \left(-x^{2} / 2\right) / \sqrt{2 \pi}\right)$, while $H_{n}(x \mid 0)=U_{n}(x / 2)$ for all $n \geqslant-1$, where $\left\{U_{n}\right\}$ are the so-called Chebyshev polynomials of the second kind, 
i.e., polynomials orthogonal with respect to the measure with the density $\sqrt{1-x^{2}} / \pi$.

Bryc in [1] has shown that there exists stationary distribution of $\mathbf{X}$ and that

$$
\forall n \in \mathbf{Z}, \quad k, i \geqslant 1: \quad \mathbf{E}\left(H_{k}\left(X_{n} \mid q\right) \mid \mathscr{F}_{\leqslant n-i}\right)=\rho^{k i} H_{k}\left(X_{n-i} \mid q\right) \quad \text { a.s. }
$$

and also that $\left\{H_{n}(x \mid q)\right\}_{n \geqslant-1}$ are orthogonal polynomials of the stationary distribution.

The case $q=-1$ is equivalent to $B=0$ and leads to marginal symmetric distribution concentrated on $\{-1,1\}$.

We will focus thus on the case $q \in(-1,1]$.

Let

$$
I_{A}(x)= \begin{cases}1 & \text { if } x \in A, \\ 0 & \text { if } x \notin A,\end{cases}
$$

denote index function of the set $A$ and define also

$$
S(q)=\left[-\frac{2}{\sqrt{1-q}}, \frac{2}{\sqrt{1-q}}\right], \quad q \in(-1,1), \quad S(1)=\mathbf{R} .
$$

It turns out that the stationary distribution of 1TSP has for $q \in(-1,1)$ the density given by

$$
f_{N}(x \mid q)=\frac{\sqrt{1-q}(q)_{\infty}}{2 \pi \sqrt{4-(1-q) x^{2}}} \prod_{k=0}^{\infty}\left(\left(1+q^{k}\right)^{2}-(1-q) x^{2} q^{k}\right) I_{S(q)}(x),
$$

while for $q=1$ it is equal to

$$
f_{N}(x \mid 1)=\frac{1}{\sqrt{2 \pi}} \exp \left(-\frac{x^{2}}{2}\right), \quad x \in \mathbf{R} .
$$

In particular for $q=0$ we have

$$
f_{N}(x \mid 0)=\frac{\sqrt{4-x^{2}}}{2 \pi} .
$$

Bryc in [1] has also found the density of the conditional distribution $X_{n} \mid X_{n-1}=y$ and later Bryc, Matysiak, and Szabłowski in [3] have found orthogonal polynomials of this conditional distribution. Namely, it turned out that this distribution has for $q \in(-1,1)$ and $y \in S(q)$ the density of the form

$$
\begin{aligned}
& f_{C N}(x \mid y, \rho, q)=\frac{\sqrt{1-q}\left(\rho^{2}, q\right)_{\infty}}{2 \pi \sqrt{4-(1-q) x^{2}}} \\
& \quad \times \prod_{k=0}^{\infty} \frac{\left(\left(1+q^{k}\right)^{2}-(1-q) x^{2} q^{k}\right)}{\left(\left(1-\rho^{2} q^{2 k}\right)^{2}-(1-q) \rho q^{k}\left(1+\rho^{2} q^{2 k}\right) x y+(1-q) \rho^{2}\left(x^{2}+y^{2}\right) q^{2 k}\right)} \\
& \quad \times I_{S(q)}(x),
\end{aligned}
$$


and that polynomials $\left\{P_{n}(x \mid y, \rho, q)\right\}_{n \geqslant-1}$ defined by

$$
\begin{aligned}
\forall n \geqslant 0: \quad P_{n+1}(x \mid y, q, \rho)= & \left(x-\rho y q^{n}\right) P_{n}(x \mid y, q, \rho) \\
& -\left(1-\rho^{2} q^{n-1}\right)[n]_{q} P_{n-1}(x \mid y q, \rho),
\end{aligned}
$$

with $P_{-1}(x \mid y, \rho, q)=0, P_{0}(x \mid y, q, \rho)=1$, are orthogonal with respect to the measure defined by the density (2.9). We will call polynomials $\left\{P_{n}\right\}$ Al-Salam-Chihara (briefly ASC).

To support intuition let us notice that both densities $f_{N}$ and $f_{C N}$ are bounded. More precisely we have the following easy remark giving bounds for the both considered densities. Let us remark also that in [18] are presented bounds for $f_{N}$ more subtle than the ones given below.

$\mathrm{R}$ e m a r k 2. i) For $|q|<1$,

$\forall x \in S(q): \quad f_{N}(x \mid q) \leqslant \frac{\sqrt{1-q}}{\pi}(q)_{\infty}(-|q|)_{\infty}^{2}, \quad$ and $\quad f_{N}\left(\frac{ \pm 2}{\sqrt{1-q}} \mid q\right)=0$,

ii) For $|q|<1$,

$$
\forall x, y \in S(q): \quad 0<C(y, \rho, q) \leqslant \frac{f_{C N}(x \mid y, \rho, q)}{f_{N}(x \mid q)} \leqslant \frac{\left(\rho^{2}\right)_{\infty}}{(\rho)_{\infty}^{4}} .
$$

Indeed, i) follows the fact that for $x \in S(q),\left(1+q^{k}\right)^{2}-(1-q) x^{2} q^{k} \leqslant$ $1+|q|^{k}$ and $\sqrt{4-x^{2}} \leqslant 2$. ii) was proved in [16, Proposition 1, vii].

Again we have two special simple cases presented in the following remark.

$\mathrm{R}$ e $\mathrm{m}$ a r $\mathrm{k} 3$. For all $n \geqslant-1$

$$
\begin{aligned}
& P_{n}(x \mid y, \rho, 1)=H_{n}\left(\frac{x-\rho y}{\sqrt{1-\rho^{2}}}\right)\left(1-\rho^{2}\right)^{n / 2}, \\
& P_{n}(x \mid y, \rho, 0)=U_{n}\left(\frac{x}{2}\right)-\rho y U_{n}\left(\frac{x}{2}\right)+\rho^{2} U_{n}\left(\frac{x}{2}\right) .
\end{aligned}
$$

There are many proofs of these simple facts. One of the simplest can be found, e.g., in [17].

We will need several properties of polynomials $\left\{H_{n}\right\}$ and $\left\{P_{n}\right\}$. Most of these properties can be found in [5] and some in [3]. We will collect these properties in the following lemma.

Lemma 1. Assume $|q|<1$. i) For $n, m \geqslant 0$ :

$$
\int_{S(q)} H_{n}(x \mid q) H_{m}(x \mid q) f_{N}(x \mid q) d x= \begin{cases}0, & n \neq m \\ {[n]_{q} !,} & n=m .\end{cases}
$$

ii) For $n \geqslant 0$ :

$$
\int_{S(q)} H_{n}(x \mid q) f_{C N}(x \mid y, \rho, q) d x=\rho^{n} H_{n}(y \mid q),
$$


iii) For $n, m \geqslant 0$ :

$$
\int_{S(q)} P_{n}(x \mid y, \rho, q) P_{m}(x \mid y, \rho, q) f_{C N}(x \mid y, \rho, q) d x= \begin{cases}0, & n \neq m \\ \left(\rho^{2}\right)_{n}[n]_{q} !, & n=m .\end{cases}
$$

iv) $\int_{S(q)} f_{C N}\left(x \mid y, \rho_{1}, q\right) f_{C N}\left(y \mid z, \rho_{2}, q\right) d y=f_{C N}\left(x \mid z, \rho_{1} \rho_{2}, q\right)$.

v) $\max _{x \in S(q)}\left|H_{n}(x \mid q)\right| \leqslant W_{n}(q) /(1-q)^{n / 2}$, where $W_{n}(q)=\sum_{i=0}^{n}\left[\begin{array}{c}n \\ i\end{array}\right]_{q}$.

vi) $\sum_{i=0}^{\infty} W_{i}(q) t^{i} /(q)_{i}=1 /(t)_{\infty}^{2}$ and $\sum_{i=0}^{\infty} W_{i}^{2}(q) t^{i} /(q)_{i}=\left(t^{2}\right)_{\infty} /(t)_{\infty}^{3}$ absolutely for $|t|,|q|<1$, where $W_{i}(q)$ is defined in $\left.\mathrm{v}\right)$.

vii) For $(1-q) x^{2} \leqslant 4$ and for all $(1-q) t^{2}<1$ :

$$
\varphi(x, t \mid q) \stackrel{\text { def }}{=} \sum_{i=0}^{\infty} \frac{t^{i}}{[i]_{q} !} H_{n}(x \mid q)=\prod_{k=0}^{\infty}\left(1-(1-q) x t q^{k}+(1-q) t^{2} q^{2 k}\right)^{-1},
$$

the convergence is absolute and uniform in $x$. Moreover $\varphi(x, t \mid q)$ is nonnegative and $\int_{S(q)} \varphi(x, t \mid q) f_{N}(x \mid q) d x=1$.

viii) For $(1-q) \max \left(x^{2}, y^{2}\right) \leqslant 4,|\rho|<1$ and for all $(1-q) t^{2}<1$ :

$$
\begin{aligned}
\tau(x, t \mid y, \rho, q) & =\sum_{i=0}^{\infty} \frac{t^{i}}{[i]_{q} !} P_{n}(x \mid y, \rho, q) \\
& =\prod_{k=0}^{\infty} \frac{\left(1-(1-q) \rho y t q^{k}+(1-q) \rho^{2} t^{2} q^{2 k}\right)}{\left(1-(1-q) x t q^{k}+(1-q) t^{2} q^{2 k}\right)}
\end{aligned}
$$

the convergence is absolute and uniform in $x$. Moreover $\tau(x, t \mid \theta, \rho, q)$ is nonnegative and $\int_{S(q)} \tau(x, t \mid y, \rho, q) f_{C N}(x \mid y, \rho, q) d x=1$.

ix) For $(1-q) \max \left(x^{2}, y^{2}\right) \leqslant 2,|\rho|<1$

$$
f_{C N}(x \mid y, \rho, q)=f_{N}(x \mid q) \sum_{n=0}^{\infty} \frac{\rho^{n}}{[n]_{q} !} H_{n}(x \mid q) H_{n}(y \mid q)
$$

and the convergence is absolute and uniform in $x$ and $y$.

P r o o f. Since the more popular are slightly modified polynomials $H_{n}$ namely the polynomials

$$
h_{n}(x \mid q)=H_{n}\left(\frac{2}{\sqrt{1-q}} \mid q\right)(1-q)^{-n / 2}, \quad x \in[-1,1]
$$

(called continuous $q$-Hermite polynomials) and

$$
p_{n}(x \mid y, \rho, q)=P_{n}\left(\frac{2 x}{\sqrt{1-q}} \mid \frac{2 y}{\sqrt{1-q}}, \rho, q\right),
$$

many properties of $q$-Hermite and Al-Salam-Chihara polynomials are formulated in terms of $h_{n}$ and $p_{n}$. 
i) It is formula 13.1 .11 of [5] with an obvious modification for the polynomials $H_{n}$ instead of $h_{n}$ and normalized weight function (i.e., $f_{N}$ ).

ii) Exercise 15.7 of [5] also in [1].

iii) Formula 15.1.5 of [5] concerns polynomials $Q_{n}(x \mid a, b, q)$ which are related to polynomials $p_{n}$ by the following relation between parameters: $a+$ $b=y \rho, a b=\rho^{2}$. Thus first we obtain formula for the polynomials $p_{n}$ and then modify it for the polynomials $P_{n}$. Accordingly we modify the weight function (i.e., $f_{C N}$ ).

iv) See $(2.6)$ of $[3]$.

v) and vi). Exercise 12.2(b) and 12.2(c) of [5].

vii)-viii) follow v) and vi). Besides nonnegativity of $\varphi$ and $\tau$ are trivial and follow formulae $1-(1-q) x t q^{k}+(1-q) t^{2} q^{2 k}=(1-q)\left(t q^{k}-x / 2\right)^{2}+1-$ $(1-q) x^{2} / 4$ and $1-(1-q) \rho y t q^{k}+(1-q) \rho^{2} t^{2} q^{2 k}=(1-q) \rho^{2}\left(q^{k} t-y /(2 \rho)\right)^{2}+$ $1-(1-q) y^{2} / 4$. Values of integrals follow i) and iii).

ix) is the famous Poisson-Mehler expansion formula. It has many proofs presented, e.g., in [5], [4], [17].

Two special cases $q=0,1$ are treated in the following remark.

$\mathrm{R} \mathrm{e} \mathrm{m} \mathrm{a} \mathrm{r} \mathrm{k} \mathrm{4.} \mathrm{Assertions} \mathrm{i)-iv)} \mathrm{and} \mathrm{ix)} \mathrm{of} \mathrm{Lemma} 1$ are true also for $q=1$. This follows elementary properties of Hermite polynomials exposed, e.g., in [6]. Further we have

$$
\begin{aligned}
\varphi(x, t \mid 1) & =\exp \left(x t-\frac{t^{2}}{2}\right), \quad \varphi(x, t \mid 0)=\frac{1}{\left(1-x t+t^{2}\right)}, \\
\tau(x, t \mid y, \rho, 1) & =\exp \left(t(x-\rho y)-\frac{t^{2}\left(1-\rho^{2}\right)}{2}\right) \\
\tau(x, t \mid y, \rho, 0) & =\frac{1-\rho y t+\rho^{2} t^{2}}{1-x t+t^{2}} .
\end{aligned}
$$

For $q=1$ we have

$$
f_{C N}(x \mid y, \rho, 1)=\frac{1}{\sqrt{2 \pi\left(1-\rho^{2}\right)}} \exp \left(-\frac{(x-\rho y)^{2}}{2\left(1-\rho^{2}\right)}\right),
$$

(i.e., Normal $N\left(\rho y, 1-\rho^{2}\right)$ distribution) while for $q=0$ we have

$$
f_{C N}(x \mid y, \rho, 0)=\frac{\left(1-\rho^{2}\right) \sqrt{4-x^{2}}}{2 \pi\left(\left(1-\rho^{2}\right)^{2}-\rho\left(1+\rho^{2}\right) x y+\rho^{2}\left(x^{2}+y^{2}\right)\right)},
$$

$x, y \in[-2,2],|\rho|<1,-$ the so-called Kesten-McKay distribution.

As mentioned earlier we are going to consider continuous time generalization of the process $\mathbf{X}$ considered in this section. More precisely, we are going to prove the existence and present some basic properties of the process $\mathbf{Y}=\left\{Y_{t}\right\}_{t \in \mathbf{R}}$, satisfying the following condition.

Condition $(\delta)$. For every positive $\delta$, random sequences $X_{n}^{(\delta)}=Y_{n \delta}$, $n \in \mathbf{Z}$, are regular 1 TSP. 
3. Auxiliary properties of one-dimensional random fields. In this section we are going to make preparations for the proof of the existence of process $\mathbf{Y}$. To do this we have to prove the following lemma.

Lemma 2. Let $\left\{X_{i}\right\}_{i \in \mathbf{Z}}$ be a 1 TSP with parameters $\rho$ and $q$. Let us fix $j \in \mathbf{N}$ and $m \in\{0, \ldots, j-1\}$ and define $Z_{k}=X_{k j+m}$ for $k \in \mathbf{Z}$. Then $\left\{Z_{k}\right\}_{k \in \mathbf{Z}}$ is also 1 TSP with parameters $\rho^{j}$ and $q$.

To do this we need generalizations of properties (2.1) and (2.2). They are given in Proposition 1 below. On the other hand, this proposition needs the following technical lemma.

Lemma 3. If $\mathbf{X}$ is a $1 T S P$, then

$$
\begin{aligned}
\mathbf{E} X_{n}^{4} & =2+q, \\
\mathbf{E} X_{n}^{2} X_{m}^{2} & =1+\rho^{2|n-m|}(1+q), \\
\mathbf{E} X_{n}^{2} X_{n-j} X_{n+k} & =\rho^{j+k} \mathbf{E} X_{n}^{4}=\rho^{j+k}(2+q) .
\end{aligned}
$$

Proposition 1. If $\mathbf{X}$ is a 1 TSP, then for $n, j, k \in \mathbf{N}$

i) $\mathbf{E}\left(X_{n} \mid \mathscr{F}_{\leqslant n-j, \geqslant n+k}\right)$ is a linear function of $X_{n-k}$ and $X_{n+j}$; more precisely we have

$$
\mathbf{E}\left(X_{n} \mid \mathscr{F}_{\leqslant n-k, \geqslant n+j}\right)=\frac{\rho^{j}\left(1-\rho^{2 k}\right)}{1-\rho^{2(j+k)}} X_{n-j}+\frac{\rho^{k}\left(1-\rho^{2 j}\right)}{1-\rho^{2(j+k)}} X_{n+k}
$$

ii) $\mathbf{E}\left(X_{n}^{2} \mid \mathscr{F}_{\leqslant n-j, \geqslant n+k}\right)$ is a linear function of $X_{n-j}^{2}, \quad X_{n+k}^{2}$, and $X_{n-j} X_{n+k} ;$ in particular,

$$
\begin{aligned}
\mathbf{E}\left(X_{n}^{2} \mid \mathscr{F}_{\leqslant n-j, \geqslant n+k}\right)= & A_{j k}^{(1)}(\rho) X_{n-j}^{2}+A_{j k}^{(2)}(\rho) X_{n+k}^{2} \\
& +B_{j k}(\rho) X_{n-j} X_{n+k}+C_{j k}(\rho),
\end{aligned}
$$

where

$$
\begin{aligned}
A_{j k}^{(1)}(\rho) & =\frac{\rho^{2 j}\left(1-\rho^{2 k}\right)\left(1-q \rho^{2 k}\right)}{\left(1-q \rho^{2(j+k)}\right)\left(1-\rho^{2(j+k)}\right)}, \\
A_{j k}^{(2)}(\rho) & =\frac{\rho^{2 k}\left(1-\rho^{2 j}\right)\left(1-q \rho^{2 j}\right)}{\left(1-q \rho^{2(j+k)}\right)\left(1-\rho^{2(j+k)}\right)}, \\
B_{j k}(\rho) & =\frac{(q+1) \rho^{j+k}\left(1-\rho^{2 j}\right)\left(1-\rho^{2 k}\right)}{\left(1-q \rho^{2(j+k)}\right)\left(1-\rho^{2(j+k)}\right)}, \\
C_{j k}(\rho) & =\frac{\left(1-\rho^{2 j}\right)\left(1-\rho^{2 k}\right)}{1-q \rho^{2(j+k)}} .
\end{aligned}
$$

Lengthy proofs of these facts as well as the proof of Lemma 2 are moved to Section 6. 


\section{4. $(\alpha, q)$ - Ornstein-Uhlenbeck and $q$-Wiener processes.}

4.1. Existence. In this subsection we are going to prove the following theorem.

Theorem 1. The $L_{2}-$ continuous $^{1)}$ and stationary process $\mathbf{Y}=$ $\left\{Y_{t}\right\}_{t \in \mathbf{R}}$ that satisfies for every $\delta>0$ Condition $(\delta)$, exists. Moreover there exist two numbers $q \in(-1,1]$ and $\alpha>0$ such that $\mathbf{Y}$ is Markov with the marginals having density $f_{N}(x \mid q)$ and the transition distribution having density $f_{C N}\left(x \mid y, e^{-\alpha|s-t|}, q\right)$ (i.e., $Y_{s} \mid Y_{t}=y \sim f_{C N}\left(x \mid y, e^{-\alpha|s-t|}, q\right)$ ).

An easy but long proof is shifted to Section 6 .

In the sequel, when considering the continuous time generalizations of 1TSP we will need the following generalization of «nonsingularity of covariance matrix» assumption considered in the case of 1TSP: Let $\mathscr{X}=\left(X_{t}\right)_{t \in \mathbf{R}}$ be square integrable stochastic process and

$$
\text { for all } n \in \mathbf{N} \text { and } 0 \leqslant t_{1}<t_{2}<\cdots<t_{n}
$$

random variables $X_{t_{1}}, \ldots, X_{t_{n}}$ are linearly independent

which we will also refer to as linear independence assumption be satisfied by $\mathscr{X}$.

4.2. $(\alpha, q)$-OU processes. Process $\mathbf{Y}$ with parameters $(\alpha, q)$ will be called continuous time $(\alpha, q)$-OU-process (OU standing for OrnsteinUhlenbeck). Let us summarize what properties of $\mathbf{Y}$ can be deduced from the properties of the discrete time regular 1TSP processes presented in [1], $[2],[7],[8],[3]$, and [15, Corollary 6, p. 13]. Some of these properties also appeared in [20] as a by-product of considering some noncommutative model or in [13] in quadratic harnesses context. Hence some of the properties are not new but they are scattered in the literature and we bring them together and collect in groups devoted to particular features of analyzed processes.

As before let us define the following $\sigma$-fields defined by $\mathbf{Y}: \mathscr{F}_{\leqslant s}:=$ $\sigma\left(X_{t}: t \leqslant s\right), \mathscr{F}_{\geqslant s}:=\sigma\left(X_{t}: t \geqslant s\right)$, and $\mathscr{F}_{\leqslant s, \geqslant t}:=\sigma\left(X_{\tau}: \tau \leqslant s \vee \tau \geqslant t\right)$ for $s<t$.

Theorem below describes the marginal and the conditional distributions, presents polynomials that are orthogonal with respect to these distributions as well as gives conditional moments with respect to one-sided ( $\mathscr{F} \leqslant s$ and $\left.\mathscr{F}_{\geqslant s}\right)$ and two-sided $\left(\mathscr{F}_{\leqslant s, \geqslant t}\right) \sigma$-fields.

Theorem 2. Let $\mathbf{Y}$ be a continuous time $(\alpha, q)$-OU-process, $-1<q \leqslant$ $1, \alpha>0$. Then its state space is $S(q)$ and the following assertions hold.

1) We have

$\forall t \in \mathbf{R}: Y_{t} \sim f_{N}(x \mid q), \quad \forall s>t: Y_{s} \mid Y_{t}=y \sim f_{C N}\left(x \mid y, e^{-\alpha(s-t)}, q\right)$.

\footnotetext{
1) $L_{2}$-continuous means mean-square continuous.
} 
2) $\mathbf{Y}$ is a stationary Markov process with $f_{C N}\left(x \mid y, e^{-\alpha(s-t)}, q\right)$ as the density of its transition probability.

3) $\mathbf{Y}$ is time symmetric. Moreover we have for any $n \in \mathbf{N}, s \in \mathbf{R}$, and $\delta, \gamma>0$

$\mathbf{E}\left(H_{n}\left(Y_{s}\right) \mid \mathscr{F}_{\leqslant s-\delta, \geqslant s+\gamma}\right)=\sum_{r=0}^{\lfloor n / 2\rfloor} \sum_{l=0}^{n-2 r} A_{r,-\lfloor n / 2\rfloor+r+l}^{(n)} H_{l}\left(Y_{s-\delta} \mid q\right) H_{n-2 r-l}\left(Y_{s+\gamma} \mid q\right)$,

where $\left\lfloor\frac{n+2}{2}\right\rfloor\left\lfloor\frac{n+3}{2}\right\rfloor$ constants $A_{r, m}^{(n)}(r=0, \ldots,\lfloor n / 2\rfloor, m=-\lfloor n / 2\rfloor+$ $r, \ldots,-\lfloor n / 2\rfloor+r+n-2 r)$ depend only on $n, q, e^{-\alpha \delta}$, and $e^{-\alpha \gamma} .^{2)}$

4) Families of polynomials $\left\{H_{n}(x \mid q)\right\}_{n \geqslant 0}$ and $\left\{P_{n}\left(x \mid y, e^{-\alpha|s-t|}, q\right)\right\}_{n \geqslant 0}$ given, respectively, by (2.5) and (2.10), are orthogonal polynomials of distributions defined by (2.7) and (2.9), respectively. That is, in particular, we have

$$
\begin{aligned}
& \forall n \geqslant 1, t \in \mathbf{R}: \quad \mathbf{E} H_{n}\left(Y_{t} \mid q\right)=0, \\
& \forall n \geqslant 1, s>t: \quad \mathbf{E}\left(P_{n}\left(Y_{s} \mid Y_{t}, e^{-\alpha(s-t)}, q\right) \mid \mathscr{F}_{\leqslant t}\right)=0 \quad \text { a.s. }
\end{aligned}
$$

5) For all $n \geqslant 1$ and $s>t$,

$$
\mathbf{E}\left(H_{n}\left(Y_{s} \mid q\right) \mid \mathscr{F}_{\leqslant t}\right)=e^{-n \alpha(s-t)} H_{n}\left(Y_{t} \mid q\right) \quad \text { a.s. }
$$

6) For all $n \geqslant 1,\left(H_{n}\left(Y_{t} \mid q\right)\right)_{t \in \mathbf{R}}$ is a stationary random process with covariance function,

$$
K_{n}(s, t)=[n]_{q} ! e^{-n \alpha|s-t|}
$$

and

as its spectral density.

$$
S_{n}(\omega \mid \alpha)=\frac{2 n \alpha[n]_{q} !}{\omega^{2}+n^{2} \alpha^{2}}
$$

Re marks concerning the proof. 1) and 2) are given in [1] and [3].

$3)$ is given in [15, Corollary 5].

4) (4.3) is given in [5] (but also in [1]) and (4.4) is given in [3].

$5)$ is given in [1].

6) Notice that from 5) it follows that for all $n, m \geqslant 1$ and $s, t \in \mathbf{R}$

$$
\mathbf{E} H_{m}\left(Y_{t} \mid q\right) H_{n}\left(Y_{s} \mid q\right)=\delta_{|n-m|} e^{-n \alpha|s-t|} \mathbf{E} H_{n}^{2}\left(Y_{t} \mid q\right)=\delta_{|n-m|} e^{-n \alpha|s-t|}[n]_{q} !,
$$

since $\mathbf{E} H_{n}^{2}\left(Y_{t} \mid q\right)=[n]_{q}$ !, by Lemma 1,i). Further we use spectral decomposition theorem.

2) Since the paper was written and submitted, the exact form of coefficients $A_{r, s}^{(n)}$ can be derived from the result presented in [16, Theorem 2] following observation that the conditional distribution of $X_{\sigma}$ given $X_{\sigma-\delta}, X_{\sigma+\gamma}$ has Askey-Wilson density with specific complex parameters. 
$\mathrm{R}$ e $\mathrm{mark} 5$. As an immediate consequence of assertion 1) of Theorem 2 we see that if $\mathbf{Y}$ is a certain $(\alpha, q)$-OU process, then the process $\mathbf{Z}$ defined by re-scaling time in the following way: $Z_{s}=Y_{\alpha s}, s \in \mathbf{R}$, is the $(1, q)$-OU process.

$\mathrm{R} \mathrm{e} \mathrm{m} \mathrm{a} \mathrm{r} \mathrm{k} \mathrm{6.} \mathrm{As} \mathrm{it} \mathrm{follows} \mathrm{from} \mathrm{assertion} \mathrm{4)} \mathrm{of} \mathrm{Theorem} \mathrm{2,} \mathrm{the}(\alpha, q)$ OU process is stationary and time homogenous. Thus its transition operator is defined by

$$
P_{s, t}(f)(y)=\int_{S(q)} f(x) f_{C N}\left(x \mid y, e^{-\alpha(t-s)}, q\right) d x
$$

for a function $f$ defined below and $t>s$, and depends in fact on $t-s=\tau$. Let us define

$$
P^{\tau}(\cdot)=P_{t, t+\tau}(\cdot)
$$

Operators $\left\{P^{\tau}\right\}_{\tau>0}$ form a semigroup of operators as it follows from Lemma 1, iv).

As conclusions of assertions of Theorem 2 we have the following theorem that contains their implications to the Markovian properties of the process $\mathbf{Y}$.

Before we formulate appropriate theorem we need to introduce some additional notation.

Let $B(q)=L_{2}\left(S(q), \mathscr{B}(S(q)), P_{N}(q)\right)$, where $\mathscr{B}(S(q))$ denotes $\sigma$-field of Borel subsets of $S(q)$ and $P_{N}(q)$ denotes measure with density $f_{N}(\cdot \mid q)$. Obviously we have

$$
B(q)=\left\{f: S(q) \rightarrow S(q): f(x)=\sum_{j=0}^{\infty} \frac{b_{j}}{\sqrt{[j]_{q} !}} H_{j}(x \mid q) ; \sum_{j \geqslant 0}\left|b_{j}\right|^{2}<\infty\right\} .
$$

Let us further denote:

$$
B^{0}(q)=\left\{f \in B(q): f(x)=\sum_{j=0}^{\infty} \frac{b_{j}}{\sqrt{[j]_{q} !}} H_{j}(x \mid q) ; \sum_{j \geqslant 1} j^{2}\left|b_{j}\right|^{2}<\infty\right\} .
$$

We have $B^{0}(q) \subset B(q)$.

Let $A$ denote infinitesimal operator of the $(\alpha, q)$-OU process.

Theorem 3. Let $\mathbf{Y}$ be $(\alpha, q)$-OU process with $|q|<1$. Then:

i) $\mathbf{D} Y_{t}=1$, and for $s \geqslant 0, \mathbf{D}\left(Y_{t+s} \mid Y_{t}\right)=1-e^{-2 \alpha s}$; hence, in particular, trajectories of $(\alpha, q)$-OU process are càdlàg functions with values in $S(q)$;

ii) transition operator $P^{\tau}$ for $\tau>0$ is defined by the following relationship:

$$
B(q) \ni f(x)=\sum_{j \geqslant 0} \frac{b_{j}}{\sqrt{[j]_{q} !}} H_{j}(x \mid q) \Rightarrow P^{\tau}(f)(x)=\sum_{j \geqslant 0} e^{-\alpha \tau j} \frac{b_{j}}{\sqrt{[j]_{q} !}} H_{j}(x \mid q) ;
$$


iii) the family of transition probabilities $\left\{P^{\tau}\right\}_{\tau>0}$ is Feller continuous; in particular, process $\mathbf{Y}$ has strong Markov property;

iv) the family $\left\{P^{\tau}\right\}_{\tau>0}$ is right-continuous, consequently process $\mathbf{Y}$ is a Feller process; moreover, its infinitesimal operator $A$ exists and is defined on the subset $B^{0}$ by the following formula:

$$
\begin{aligned}
B^{0}(q) & \ni f(x)=\sum_{j \geqslant 0} \frac{b_{j}}{\sqrt{[j]_{q} !}} H_{j}(x \mid q) \\
& \Rightarrow \quad A(f)(x)=-\alpha \sum_{j \geqslant 1} \frac{j b_{j}}{\sqrt{[j]_{q} !}} H_{j}(x \mid q) \in B(q) .
\end{aligned}
$$

The proof is shifted to Section 6 .

$\mathrm{Re} \mathrm{mark} 7$. Let us remark that recently Anshelevich in [22, Lemma 20] expressed the infinitesimal operator in an integral form. To be precise he expressed in this form the infinitesimal operator of the socalled $q$-Wiener ( $q$-Brownian motion as he calls it) process to be considered in the next subsection and related to $(\alpha, q)$-OU by the continuous transformation (4.9).

The detailed forms of constants $A_{r, m}^{(n)}$ defined by (4.5) are given below following Corollary 6 of [15].

\section{Corollary 1.}

$$
A_{0,-\lfloor n / 2\rfloor+l}^{(n)}=\left[\begin{array}{c}
n \\
l
\end{array}\right]_{q} \frac{e^{-(n-l) \alpha \delta}\left(e^{-2 \alpha \gamma}\right)_{n-l} e^{-l \alpha \gamma}\left(e^{-2 \alpha \delta}\right)_{l}}{\left(e^{-2 \alpha(\delta+\gamma)}\right)_{n}},
$$

$l=0, \ldots, n, n=1, \ldots, 4$.

If $n \leqslant 3$, then

$$
A_{1,-\lfloor n / 2\rfloor+l}^{(n)}=-[n-1]_{q} e^{-\alpha(\delta+\gamma)} A_{0,-\lfloor n / 2\rfloor+l}^{(n)}, \quad l=1, \ldots, n-1 .
$$

If $n=4$, then

$$
\begin{aligned}
& A_{1, j}^{(4)}=-[3]_{q} e^{-\alpha(\delta+\gamma)} A_{0, j}^{(4)}, \quad j=-1,1, \\
& A_{1,0}^{(4)}=-[2]_{q}^{2} e^{-\alpha(\delta+\gamma)} A_{0,0}^{(4)}, \\
& A_{2,0}^{(4)}=q(1+q) e^{-2 \alpha(\delta+\gamma)} A_{0,0}^{(4)} .
\end{aligned}
$$

In particular, we obtain known (see, e.g., [9]) formula

$$
\begin{aligned}
\mathbf{D} & \left(Y_{s} \mid \mathscr{F}_{\leqslant s-\delta, \geqslant s+\gamma}\right)=\frac{\left(1-e^{-2 \alpha \delta}\right)\left(1-e^{-2 \alpha \gamma}\right)}{1-q e^{-2 \alpha(\delta+\gamma)}} \\
& \times\left(1-\frac{(1-q)\left(Y_{s-\delta}-e^{-\alpha(\delta+\gamma)} Y_{s+\gamma}\right)\left(Y_{s+\gamma}-e^{-\alpha(\delta+\gamma)} Y_{s-\delta}\right)}{\left(1-e^{-2 \alpha(\delta+\gamma)}\right)^{2}}\right) .
\end{aligned}
$$

Following assertion vii) of Lemma 1 we can express assertion 5) of Theorem 2 in the following martingale-like form. 
Theorem 4. For $|q|<1$ we have

$$
\forall \gamma^{2}(1-q)<1, s>t: \quad \mathbf{E}\left(\varphi\left(Y_{s}, \gamma \mid q\right) \mid \mathscr{F} \leqslant t\right)=\varphi\left(Y_{t}, \gamma e^{-\alpha(s-t)} \mid q\right) \quad \text { a.s. }
$$

where positive function $\varphi$ is defined in Lemma 1, vii).

In particular, for $q=0$ (so called free $\alpha$-OU-process) we have

$$
\begin{aligned}
\forall|\gamma|<1, s>t: \quad & \mathbf{E}\left(\frac{1}{1-\gamma Y_{s}+\gamma^{2}} \mid \mathscr{F}_{\leqslant t}\right) \\
& =\frac{1}{1-\gamma e^{-\alpha(t-s)} Y_{t}+\gamma^{2} e^{-2 \alpha(t-s)}} \quad \text { a.s. }
\end{aligned}
$$

while for $q=1$ we get well-known formula:

$$
\begin{aligned}
\forall \gamma & =\mathbf{R}, s>t: \quad \mathbf{E}\left(\exp \left(\gamma Y_{s}-\frac{\gamma^{2}}{2}\right) \mid \mathscr{F}_{\leqslant t}\right) \\
& =\exp \left(\gamma e^{-\alpha(s-t)} Y_{t}-\frac{1}{2}\left(\gamma e^{-\alpha(s-t)}\right)^{2}\right) \quad \text { a.s. }
\end{aligned}
$$

Strict proof is very much alike the proof of Corollary 4 below, so we will not present it here.

$\mathrm{R}$ e $\mathrm{m}$ a $\mathrm{rk} 8$. Notice that following considerations of Section 2, concerning existence of the $(\alpha, q)$-OU process one needed only the following two, symmetric in time, conditions apart from the linear independence:

1) for all $n \in \mathbf{Z}$ and $d>0$,

$$
\mathbf{E}\left(Y_{n d} \mid \mathscr{F}_{\leqslant(n-1) d, \geqslant(n+1) d}\right)=\frac{e^{-\alpha d}}{1+e^{-2 \alpha d}}\left(Y_{(n-1) d}+Y_{(n+1) d}\right),
$$

2) for all $n \in \mathbf{Z}$ and $d>0$,

$$
\mathbf{E}\left(Y_{n d}^{2} \mid \mathscr{F}_{\leqslant(n-1) d, \geqslant(n+1) d}\right)=\widehat{A}\left(Y_{(n-1) d}^{2}+Y_{(n+1) d}^{2}\right)+\widehat{B} Y_{(n-1) d} Y_{(n+1) d}+\widehat{C},
$$

where $\widehat{A}_{11}^{(1)}\left(e^{-\alpha d}\right)=A_{11}^{(2)}\left(e^{-\alpha d}\right) \widehat{B}=B_{11}\left(e^{-\alpha d}\right), \widehat{C}=C_{11}\left(e^{-\alpha d}\right)$. That is, we need only symmetric (and discrete for all increments $d>0$ ) versions of condition defining $\mathbf{E}\left(Y_{s}^{2} \mid \mathscr{F}_{\leqslant s-\delta, \geqslant s+\gamma}\right)$.

$\mathrm{R}$ e $\mathrm{m}$ a r k 9. Notice that continuous time $(\alpha, q)$-OU process does not exist for $q>1$. It is so because for discrete time 1TSP with parameters $(q, \rho)$ the following relationship between parameters $q$ and $\rho$ must be satisfied for some integer $n: \rho^{2} q^{n}=1$ or equivalently the ratio $\log q / \log \rho^{2}$ must be equal to some integer. However if $(\alpha, q)$-OU process existed, then parameter $\rho$ would depend on time parameter $t$ in the following way: $\rho^{2}=\exp (-2 \alpha t)$ for some fixed positive $\alpha$ and consequently $(\log q) /(\alpha t)$ would have to be integer for all real $t$ which is impossible. 
4.3. $q$-Wiener process. Let $\mathbf{Y}$ be a given $(\alpha, q)$-OU process. Let us define:

$$
X_{0}=0, \quad \forall \tau>0: \quad X_{\tau}=\sqrt{\tau} Y_{(\log \tau) /(2 \alpha)} .
$$

Process $\mathbf{X}=\left(X_{\tau}\right)_{\tau \geqslant 0}$ will be called $q$-Wiener process. Let us also introduce the following filtration:

$$
\mathscr{F}_{\leqslant \theta}^{X}=\sigma\left(X_{\tau}: \tau \leqslant \theta\right)=\sigma\left(Y_{t}: t \leqslant \frac{\log \theta}{2 \alpha}\right)\left(=\mathscr{F}_{\leqslant(\log \theta) /(2 \alpha)}\right) .
$$

$\mathrm{R}$ e $\mathrm{m}$ a r k 10. From (4.9) and from the properties of $(\alpha, q)$-OU process it follows that $\mathbf{X}$ is a self-similar process, since for $c>0$ we see that $\left\{Z_{\tau}\right\}_{\tau \geqslant 0}$, where $Z_{\tau}=c^{-1} X_{c^{2} \tau}, \tau \geqslant 0$, is also a $q$-Wiener process.

Following definition given by (4.9) and Theorem 2 of the previous section we have the following theorem whose detailed proof is simple but lengthy and thus is shifted to Section 6 .

Theorem 5. Let $\mathbf{X}$ be a $q$-Wiener process, then it has the following properties.

1) $\forall \tau, \sigma \geqslant 0: \operatorname{cov}\left(X_{\tau}, X_{\sigma}\right)=\min (\tau, \sigma)$.

2) $\forall \tau>0: X_{\tau} \sim(1 / \sqrt{\tau}) f_{N}(x / \sqrt{\tau} \mid q)$. $\sqrt{\sigma / \tau}, q)$.

3) For $\tau>\sigma: X_{\tau}-X_{\sigma} \mid X_{\sigma}=y \sim(1 / \sqrt{\tau}) f_{C N}((x+y) / \sqrt{\tau} \mid y / \sqrt{\sigma}$,

4) For all $n \geqslant 1$ and $0<\sigma \leqslant \tau$ we have

$$
\begin{aligned}
\mathbf{E}\left(\tau^{n / 2} H_{n}\left(\frac{X_{\tau}}{\sqrt{\tau}} \mid q\right) \mid \mathscr{F}_{\leqslant \sigma}^{X}\right) & =\sigma^{n / 2} H_{n}\left(\frac{X_{\sigma}}{\sqrt{\sigma}} \mid q\right) \quad \text { a.s. }, \\
\mathbf{E}\left(\sigma^{-n / 2} H_{n}\left(\frac{X_{\sigma}}{\sqrt{\sigma}} \mid q\right) \mid \mathscr{F}_{\geqslant \tau}^{X}\right) & =\tau^{-n / 2} H_{n}\left(\frac{X_{\tau}}{\sqrt{\tau}} \mid q\right) \quad \text { a.s. }
\end{aligned}
$$

Hence for all $n \geqslant 1$ the pair $\left(Z_{\tau}^{(n)}, \mathscr{F}_{\leqslant \tau}^{X}\right)_{\tau \geqslant 0}$, where $Z_{\tau}^{(n)}=\tau^{n / 2} H_{n}\left(X_{\tau} / \sqrt{\tau} \mid q\right)$, $\tau \geqslant 0$, is a martingale and the pair $\left(V_{\tau}^{(n)}, \mathscr{F}_{\geqslant}^{X}\right)_{\tau \geqslant 0}$, where $V_{\tau}^{(n)}=$ $\tau^{-n / 2} H_{n}\left(X_{\tau} / \sqrt{\tau} \mid q\right)$, is the reverse martingale. In particular $\mathbf{X}$ is a martingale and $\left(X_{\tau} / \tau, \mathscr{F} \geqslant \tau\right)_{\tau \geqslant 0}$ is the reversed martingale.

5) We have also for all $n \geqslant 1, \delta, \gamma \geqslant 0, \sigma \geqslant \delta$

$$
\begin{aligned}
\mathbf{E}( & \left.H_{n}\left(\frac{X_{\sigma}}{\sqrt{\sigma}} \mid q\right) \mid \mathscr{F}_{\leqslant \sigma-\delta, \geqslant \sigma+\gamma}\right) \\
& =\sum_{r=0}^{\lfloor n / 2\rfloor} \sum_{l=0}^{n-2 r} A_{r,-\lfloor n / 2\rfloor+r+l}^{(n)} H_{l}\left(\frac{X_{\sigma-\delta}}{\sqrt{\sigma-\delta}} \mid q\right) H_{n-2 r-l}\left(\frac{X_{\sigma+\gamma}}{\sqrt{\sigma+\gamma}} \mid q\right),
\end{aligned}
$$

where $\left\lfloor\frac{n+2}{2}\right\rfloor\left\lfloor\frac{n+3}{2}\right\rfloor$ constants $A_{r, s}^{(n)}(r=0, \ldots,\lfloor n / 2\rfloor, s=-\lfloor n / 2\rfloor+$ $r, \ldots,-\lfloor n / 2\rfloor+r+n-2 r)$ depend only on $n, q$, and numbers $\sigma, \delta$, and $\gamma \cdot{ }^{3}$ )

3) See footnote ${ }^{2)}$ to assertion 3) of Theorem 2. 
We have the following immediate, easy observation.

$\mathrm{R}$ e $\mathrm{m}$ a r k 11 . From assertion 2 ) of Theorem 5 we see that for every $t>0, t X_{1 / t}$ and $X_{t}$ have the same distribution.

Corollary 2. Let $\mathbf{X}$ be a $q$-Wiener process. Then the following assertion hold.

i) We have

$$
\begin{aligned}
\forall \tau>\sigma>0: & \mathbf{E}\left(\left(X_{\tau}-X_{\sigma}\right)^{2} \mid \mathscr{F}_{\leqslant \sigma}^{X}\right)=\tau-\sigma \quad \text { a.s. } \\
\forall \tau>\sigma>0: & \mathbf{E}\left(\left(X_{\tau}-X_{\sigma}\right)^{3} \mid \mathscr{F}_{\leqslant \sigma}^{X}\right)=-(1-q)(\tau-\sigma) X_{\sigma} \quad \text { a.s. } \\
\forall \tau>\sigma>0: & \mathbf{E}\left(\left(X_{\tau}-X_{\sigma}\right)^{4} \mid \mathscr{F}_{\leqslant \sigma}^{X}\right)= \\
& =(\tau-\sigma)\left(X_{\sigma}^{2}(1-q)^{2}+(2+q)(\tau-\sigma)+\sigma\left(1-q^{2}\right)\right) \quad \text { a.s. }
\end{aligned}
$$

Hence $\left\langle X_{\tau}\right\rangle=\tau$, moreover $\mathbf{X}$ does not have independent increments.

ii) Almost every path of the process $\mathbf{X}$ has at any point left- and righthand side limits, thus $\mathbf{X}$ can be modified to have càdlàg trajectories.

iii) Process $\mathbf{X}$ is Feller continuous process and has strong Markov property.

The following corollary gives more detailed consequences of [15, Corollary 6, p. 13]. These conditional moments are known of course (see, e.g., [13] for $n=1,2$ ) since $q$-Wiener process, as pointed out in the introduction can be obtained as a particular quadratic harness.

Corollary 3. We have

$$
A_{0,-\lfloor n / 2\rfloor+l}^{(n)}=\left[\begin{array}{c}
n \\
l
\end{array}\right]_{q} \frac{\rho_{1}^{n-l}\left(\rho_{2}^{2}\right)_{n-l} \rho_{2}^{l}\left(\rho_{1}^{2}\right)_{l}}{\left(\rho_{1}^{2} \rho_{2}^{2}\right)_{n}}, \quad l=0, \ldots, n, \quad n=1, \ldots, 4,
$$

where $\rho_{1}=\sqrt{(\sigma-\delta) / \sigma}, \rho_{2}=\sqrt{\sigma /(\sigma+\gamma)}$.

If $n \leqslant 3$, then

$$
A_{1,-\lfloor n / 2\rfloor+l}^{(n)}=-[n-1]_{q} \rho_{1} \rho_{2} A_{0,-\lfloor n / 2\rfloor+l}^{(n)}, \quad l=1, \ldots, n-1,
$$

If $n=4$, then

$$
\begin{aligned}
& A_{1, j}^{(4)}=-[3]_{q} \rho_{1} \rho_{2} A_{0, j}^{(4)}, \quad j=-1,1, \\
& A_{1,0}^{(4)}=-[2]_{q}^{2} \rho_{1} \rho_{2} A_{0,0}^{(4)}, \\
& A_{2,0}^{(4)}=q(1+q) \rho_{1}^{2} \rho_{2}^{2} A .
\end{aligned}
$$

In particular, we can deduce the following known (from, say, [20], [10] or [9]) formulae for $0 \leqslant \delta \leqslant \sigma$ and $\gamma \geqslant 0$ :

$$
\mathbf{E}\left(X_{\sigma} \mid \mathscr{F}_{\leqslant \sigma-\delta, \geqslant \sigma+\gamma}\right)=\frac{\gamma}{\delta+\gamma} X_{\sigma-\delta}+\frac{\delta}{\delta+\gamma} X_{\sigma+\gamma} \quad \text { a.s. },
$$




$$
\begin{aligned}
& \mathbf{E}\left(X_{\sigma}^{2} \mid \mathscr{F}_{\leqslant \sigma-\delta, \geqslant \sigma+\gamma}\right)=\frac{\delta \gamma}{(\delta+\gamma)(\sigma(1-q)+\gamma+q \delta)} \\
& \times\left(((1-q) \sigma+\gamma) \frac{X_{\sigma-\delta}^{2}}{\delta}+((1-q) \sigma+q \delta) \frac{X_{\sigma+\gamma}^{2}}{\gamma}\right. \\
& \left.\quad+(q+1) X_{\sigma-\delta} X_{\sigma+\gamma}+(\delta+\gamma)\right),
\end{aligned}
$$

and

$$
\begin{aligned}
& \mathbf{D}\left(X_{\sigma} \mid \mathscr{F}_{\leqslant \sigma-\delta, \geqslant \sigma+\gamma}\right)=\frac{\delta \gamma}{\sigma(1-q)+\gamma+q \delta} \\
& \quad \times\left(1-(1-q) \frac{X_{\sigma+\gamma}-X_{\sigma-\delta}}{\delta+\gamma} \frac{(\sigma+\gamma) X_{\sigma-\delta}-(\sigma-\delta) X_{\sigma+\gamma}}{\delta+\gamma}\right) .
\end{aligned}
$$

The following assertion is a consequence of Theorem 5 .

Corollary 4. Let $\mathbf{X}=\left(X_{\tau}\right)_{\tau \geqslant 0}$ be a $q$-Wiener process. Then for all $s \in \mathbf{R}$, the pairs

$$
\begin{aligned}
& \left(\varphi\left(\frac{X_{\tau}}{\sqrt{\tau}}, s \sqrt{\tau} \mid q\right), \mathscr{F}_{\leqslant \tau}^{X}\right)_{1 /\left((1-q) s^{2}\right)>\tau \geqslant 0}, \\
& \left(\varphi\left(\frac{X_{\sigma}}{\sqrt{\sigma}}, \frac{s}{\sqrt{\sigma}} \mid q\right), \mathscr{F}_{\geqslant \sigma}^{X}\right)_{\sigma>(1-q) s^{2} \geqslant 0}
\end{aligned}
$$

are positive, respectively, martingale and reversed martingale, where function $\varphi(x, t \mid q)$ is a characteristic function of q-Hermite polynomials and is defined in Lemma 1, vii).

In particular, we get:

$$
\begin{aligned}
\forall s \in \mathbf{R}, \frac{1}{(1-q) s^{2}}>\tau \geqslant 0: & \mathbf{E}\left(\varphi\left(\frac{X_{\tau}}{\sqrt{\tau}}, s \sqrt{\tau} \mid q\right)\right)=1, \\
\forall s \in \mathbf{R}, s^{2}(1-q)<\tau: & \mathbf{E}\left(\varphi\left(\frac{X_{\tau}}{\sqrt{\tau}}, \frac{s}{\sqrt{\tau}} \mid q\right)\right)=1 .
\end{aligned}
$$

0-Wiener process (sometimes called free Wiener process) satisfies:

$$
\begin{array}{ll}
\forall s \in \mathbf{R}, 0 \leqslant \sigma<\tau<\frac{1}{s^{2}}: \quad & \mathbf{E}\left(\frac{1}{1-s X_{\tau}+\tau s^{2}} \mid \mathscr{F}_{\leqslant \sigma}^{X}\right) \\
& =\frac{1}{1-s X_{\sigma}+\sigma s^{2}} \quad \text { a.s. }, \\
\forall s \in \mathbf{R}, 0<s^{2}<\sigma<\tau: & \mathbf{E}\left(\frac{1}{1-s X_{\sigma} / \sigma+s^{2} / \sigma} \mid \mathscr{F}_{\geqslant \tau}^{X}\right) \\
& =\frac{1}{1-s X_{\tau} / \tau+s^{2} / \tau} \quad \text { a.s. }
\end{array}
$$


1-Wiener process satisfies of course, for $\sigma>\tau \geqslant 0$,

$$
\begin{aligned}
& \forall s \in \mathbf{R}: \quad \mathbf{E}\left(\exp \left(s X_{\tau}-\frac{\tau s^{2}}{2}\right) \mid \mathscr{F}_{\leqslant \sigma}^{X}\right)=\exp \left(s X_{\sigma}-\frac{\sigma s^{2}}{2}\right) \quad \text { a.s. }, \\
& \forall s \in \mathbf{R}: \quad \mathbf{E}\left(\exp \left(\frac{s X_{\sigma}}{\sigma}-\frac{s^{2}}{2 \sigma}\right) \mid \mathscr{F}_{\geqslant \tau}^{X}\right)=\exp \left(\frac{s X_{\tau}}{\tau}-\frac{s^{2}}{2 \tau}\right) \quad \text { a.s. }
\end{aligned}
$$

$\mathrm{R}$ e $\mathrm{m}$ a r k 12. Let us recall following [13] that quadratic harnesses are roughly speaking square integrable processes $\left\{Z_{t}\right\}_{t \in \mathbf{R}_{+}}$that satisfy 5 conditions imposed on their covariance functions and on the first two conditional moments given the past and given the past and the future. These conditions require that $\mathbf{E}\left(Z_{t} \mid \mathscr{F}_{\leqslant t-\delta}\right)$ and $\mathbf{E}\left(Z_{t} \mid \mathscr{F}_{\leqslant t-\delta, \geqslant t+\gamma}\right)$ be linear functions while $\mathbf{E}\left(Z_{t}^{2} \mid \mathscr{F}_{\leqslant t-\delta}\right)$ and $\mathbf{E}\left(Z_{t}^{2} \mid \mathscr{F}_{\leqslant t-\delta, \geqslant t+\gamma}\right)$ be a quadratic functions of $Z_{t-\delta}$ and $Z_{t+\gamma}$, respectively. Examining assertion of Corollary 3 we see that $q$-Wiener process has this property. Thus we deduce that $\mathbf{X}$ is a quadratic harness with parameters (introduced in [13]) $\theta=\eta=\tau=\sigma=0$.

$\mathrm{R}$ e mark 13. From assertion 4) of Theorem 5 it follows that $\left(X_{\tau}, \mathscr{F}_{\leqslant \tau}^{X}\right)_{\tau \geqslant 0}, \quad\left(X_{\tau}^{2}-\tau, \mathscr{F}_{\leqslant \tau}^{X}\right)_{\tau \geqslant 0} \quad$ are martingales and $\left(X_{\tau} / \tau, \mathscr{F}_{\geqslant \tau}^{X}\right)_{\tau>0}$, $\left(X_{\tau}^{2} / \tau^{2}-1 / \tau, \mathscr{F}_{\geqslant \tau}^{X}\right)_{\tau>0}$ are reversed martingales. Thus if the main result of Wesołowski's paper [19] (stating that a process satisfying these conditions should be a Wiener process) was true we would deduce that $\mathbf{X}$ is the Wiener process. But it is not true at least for $|q|<1$. Let us note that Wesolowski is aware of this, since in [9] he (together with Bryc) gives examples that contradict the result of [19]. He however did not point out where was the mistake. Recall that Wesołowski considered processes that have the property that they and their squares were both martingales and reversed martingales. Wesołowski's argument was based on the value $\mathbf{E}\left(X_{\tau}-X_{\sigma}\right)^{4}$ that had to be calculated for the considered process. Following formulae presented above we deduce that $\mathbf{E}\left(X_{\tau}-X_{\sigma}\right)^{4}=(2+q)(\tau-\sigma)^{2}+2(1-q) \sigma(\tau-\sigma)$ for a $q$-Wiener process, while Wesolowski stated that $\mathbf{E}\left(X_{\tau}-X_{\sigma}\right)^{4}=$ $c(\tau-\sigma)^{2}+2(c-3) \sigma(\sigma+\tau)$, where $c=\mathbf{E} X_{\tau}^{4} / \tau^{2}$. In the case of $q$-Wiener process one can calculate that $c=2+q$, thus Wesołowski made almost trivial mistake in his calculations.

$\mathrm{R} \mathrm{e} \mathrm{m} \mathrm{a} \mathrm{r} \mathrm{k} \mathrm{14.} \mathrm{Following} \mathrm{(4.12)} \mathrm{and} \mathrm{(4.13)} \mathrm{we} \mathrm{have:} \mathrm{for} \mathrm{all} 0<\sigma<$ $\tau \leqslant v<\omega$

$$
\begin{aligned}
\operatorname{cov}\left(X_{\tau}-X_{\sigma}, X_{\omega}-X_{v}\right)= & 0 \\
\operatorname{cov}\left(\left(X_{\tau}-X_{\sigma}\right)^{2},\left(X_{\omega}-X_{v}\right)^{2}\right)= & 0 \\
\operatorname{cov}\left(\left(X_{\tau}-X_{\sigma}\right)^{3},\left(X_{\omega}-X_{v}\right)^{3}\right)= & -(1-q)(\tau-\sigma)(\omega-v) \\
& \times(\tau(2+q)-\sigma(1+2 q)) .
\end{aligned}
$$

5. Open problems. As it follows from the description of $q$-Wiener and $(\alpha, q)$-OU processes they do not allow continuous paths modifications. 
Their paths have jumps. Besides both left- and right-hand side limits exist at any jumping point. Consequently the paths of these processes do not have discontinuities of the second kind. Thus there are several immediate questions:

1) In general on every finite interval there can be infinitely many jumps. Is it true? Or can one prove some additional properties of these processes that would eliminate this case? Certainly such properties do not exist for all $|q|<1$. But may be one can find $q_{0}$ such that for $q_{0}<q<1$ the $q$-Wiener process has only finite number of jumps on every finite interval?

2 ) What is the distribution of the size of jumps of $(\alpha, q)$-OU process. It is stationary. But is it of continuous, discrete or singular type? Or maybe it is a mixture?

3) On the other hand, for every $\varepsilon>0$ there are only finite number of jumps of the size not less than $\varepsilon$. It follows from the symmetry of $(\alpha, q)$-OU processes with respect to the time argument that inter jumps intervals between such jumps have the same distributions. What is the distribution of the length of those intervals. Strong Markov property would suggest exponential distribution. Is it true? Do they form a renewal process, i.e., are those intervals independent? Probably not, but it needs justification.

4) What are the properties of quadratic variations of the martingales associated with the $q$-Wiener processes?

5) Recently Anshelevich et al. (see [12]) have proved so called free infinite divisibility of $q$-Normal distribution. Is transitional distribution with density $f_{C N}$ also free infinitely divisible?

6. Proofs of the results. $\mathrm{P} \mathrm{r}$ o o f of $\mathrm{L} \mathrm{e} \mathrm{m} \mathrm{m}$ a 3 . Remembering that $H_{4}(x \mid q)=x^{4}-\left(3+2 q+q^{2}\right) x^{2}+\left(1+q+q^{2}\right)$ we have

$$
x^{4}=H_{4}(x \mid q)+\left(3+2 q+q^{2}\right) H_{2}(x \mid q)+2+q .
$$

Hence $\mathbf{E} X_{n}^{4}=2+q$, since $\mathbf{E} H_{4}\left(X_{m}\right)=\mathbf{E} H_{2}\left(X_{m}\right)=0$. Now using the fact that $\mathbf{E}\left(H_{2}\left(X_{n}\right) \mid \mathscr{F}_{\leqslant m}\right)=\rho^{2(n-m)} H_{2}\left(X_{m}\right)$ for $m<n$ we get:

$$
\begin{aligned}
\mathbf{E} X_{n}^{2} X_{m}^{2} & =\mathbf{E} X_{m}^{2}\left(H_{2}\left(X_{n} \mid q\right)+1\right)=1+\mathbf{E}\left(X_{m}^{2} \mathbf{E}\left(H_{2}\left(X_{n} \mid q\right) \mid \mathscr{F}_{\leqslant m}\right)\right) \\
& =1+\rho^{2|n-m|} \mathbf{E} X_{m}^{2} H_{2}\left(X_{m}\right)=1+\rho^{2|n-m|} \mathbf{E}\left(X_{m}^{4}-X_{m}^{2}\right) \\
& =1+\rho^{2|n-m|}(2+q-1) .
\end{aligned}
$$

To get (3.1c), we calculate:

$$
\begin{aligned}
\mathbf{E} X_{n}^{2} X_{n-j} X_{n+k} & =\mathbf{E}\left(X_{n}^{2} X_{n-j} \mathbf{E}\left(X_{n+k} \mid \mathscr{F}_{\leqslant n}\right)\right)=\rho^{k} \mathbf{E} X_{n}^{3} X_{n-j} \\
& =\rho^{k+j} \mathbf{E}\left(X_{n}^{3} \mathbf{E}\left(X_{n-j} \mid \mathscr{F}_{\geqslant n}\right)\right)=\rho^{k+j} \mathbf{E} X_{n}^{4}=\rho^{k+j}(2+q) .
\end{aligned}
$$

Lemma 3 is proved. 
$\mathrm{Pr}$ o of of Proposition 1 . For fixed natural numbers $k, j$ let us denote $Z_{i}=\mathbf{E}\left(X_{n+i} \mid \mathscr{F}_{\leqslant n-k, \geqslant n+j}\right)$. Let $\mathbf{Z}=\left[Z_{i}\right]_{i=-k+1}^{j-1}$. Now notice that for each coordinate of the vector $\mathbf{Z}$ we have:

$$
\begin{aligned}
Z_{i} & =\mathbf{E}\left(X_{i} \mid \mathscr{F}_{\leqslant n-k, \geqslant n+j}\right)=\mathbf{E}\left(\mathbf{E}\left(X_{i} \mid \mathscr{F}_{\neq i}\right) \mid \mathscr{F}_{\leqslant n-k, \geqslant n+j}\right) \\
& =\frac{\rho}{1+\rho^{2}}\left(\mathbf{E}\left(X_{i-1} \mid \mathscr{F}_{\leqslant n-k, \geqslant n+j}\right)+\mathbf{E}\left(X_{i+1} \mid \mathscr{F}_{\leqslant n-k, \geqslant n+j}\right)\right) \\
& = \begin{cases}\frac{\rho}{1+\rho^{2}}\left(Z_{i-1}+Z_{i+1}\right) & \text { if } i \neq-k+1 \vee j-1, \\
\frac{\rho}{1+\rho^{2}} X_{n-k}+\frac{\rho}{1+\rho^{2}} Z_{-k+2} & \text { if } i=-k+1, \\
\frac{\rho}{1+\rho^{2}} X_{n+j}+\frac{\rho}{1+\rho^{2}} Z_{j-2} & \text { if } i=j-1 .\end{cases}
\end{aligned}
$$

Hence we have a vector linear equation

$$
\mathbf{Z}=A Z+J,
$$

where

$$
\begin{aligned}
\mathbf{A} & =\left[\begin{array}{cccc}
0 & \frac{\rho}{1+\rho^{2}} & \ldots & 0 \\
\frac{\rho}{1+\rho^{2}} & 0 & \ldots & 0 \\
\cdots & \ldots & \ldots & \frac{\rho}{1+\rho^{2}} \\
0 & 0 & \ldots & 0
\end{array}\right], \\
\mathbf{J}^{T} & =\left[\begin{array}{ccc}
\rho \\
\frac{\rho}{1+\rho^{2}} X_{n-k}, 0, \ldots, 0, \frac{\rho}{1+\rho^{2}} X_{n+j}
\end{array}\right] .
\end{aligned}
$$

Now notice that the matrix $\mathbf{I}-A$, where $\mathbf{I}$ denotes unity matrix is nonsingular. This is so because sum of the absolute values of elements in each row of the matrix $\mathbf{A}$ is less than 1 which means that eigenvalues of the matrix $\mathbf{I}-\mathbf{A}$ are inside circle at center in 1 and radius less than 1 , thus nonzero. Consequently each component of the vector $\mathbf{Z}$ is a linear function of $X_{n-k}$ and $X_{n+j}$. Having linearity of $\mathbf{E}\left(X_{i} \mid \mathscr{F}_{\leqslant n-k, \geqslant n+j}\right)$ with respect to $X_{n-k}$ and $X_{n+j}$ we get (3.2).

Let us denote $m_{i, j}=\mathbf{E}\left(X_{n+i} X_{n+j} \mid \mathscr{F}_{\leqslant n-l, \geqslant n+k}\right)$, for $i, j=-l,-l+$ $1, \ldots, k-1, k$. Notice that using $(3.2)$ we get $m_{i, j}=m_{j, i}$ and that

$$
\begin{aligned}
m_{-l, j} & =X_{n-l} \mathbf{E}\left(X_{n+j} \mid \mathscr{F}_{\leqslant n-l, \geqslant n+k}\right) \\
& =\frac{\rho^{l+j}\left(1-\rho^{2 k-2 j}\right)}{1-\rho^{2(k+l)}} X_{n-l}^{2}+\frac{\rho^{k-j}\left(1-\rho^{2 l+2 j}\right)}{1-\rho^{2(k+l)}} X_{n-l} X_{n+k}, \\
m_{k, j} & =X_{n+k} \mathbf{E}\left(X_{n+j} \mid \mathscr{F}_{n-l \leqslant, \geqslant n+k}\right) \\
& =\frac{\rho^{l+j}\left(1-\rho^{2 k-2 j}\right)}{1-\rho^{2(k+l)}} X_{n-l} X_{n+k}+\frac{\rho^{k-j}\left(1-\rho^{2 l+2 j}\right)}{1-\rho^{2(k+l)}} X_{n+k}^{2},
\end{aligned}
$$


$m_{-l,-l}=X_{n-1}^{2}, m_{k, k}=X_{n+k}^{2}$. Besides we have for $i, j=-j+1, \ldots, k-1$ and $i \neq j$

$$
\begin{aligned}
m_{i, j} & =\mathbf{E}\left(X_{n+i} X_{n+j} \mid \mathscr{F}_{\leqslant n-l, \geqslant n+k}\right)=\mathbf{E}\left(X_{n+i} \mathbf{E}\left(X_{n+j} \mid \mathscr{F}_{\neq n+j}\right) \mid \mathscr{F}_{n-l \leqslant, \geqslant n+k}\right) \\
& =\frac{\rho}{1+\rho^{2}}\left(m_{i, j-1}+m_{i, j+1}\right)
\end{aligned}
$$

and if $i=j$, then

$$
\begin{aligned}
m_{i, i} & =\mathbf{E}\left(X_{n+i}^{2} \mid \mathscr{F}_{\leqslant n-l, \geqslant n+k}\right)=\mathbf{E}\left(\mathbf{E}\left(X_{n+i}^{2} \mid \mathscr{F}_{\neq n+i}\right) \mid \mathscr{F}_{n-l \leqslant, \geqslant n+k}\right) \\
& =A\left(m_{i-1, i-1}+m_{i+1, i+1}\right)+B m_{i-1, i+1}+C .
\end{aligned}
$$

Notice also that we have in fact $(l+k-2)^{2}$ unknowns and $(l+k-2)^{2}$ linear equations. Moreover random variables $m_{i, j}$ are well defined, since conditional expectation is uniquely defined (up to set of probability 1 ). Thus we get the main assertion of the proposition. Now we know that for some $A_{j}, B_{j}, C_{j}$ we have

$$
\mathbf{E}\left(X_{n}^{2} \mid \mathscr{F}_{n-j \leqslant, \geqslant n+k}\right)=A_{j k}^{(1)} X_{n-j}^{2}+A_{j k}^{(2)} X_{n+k}^{2}+B_{j k} X_{n-j} X_{n+k}+C_{j k} .
$$

First thing to notice is that (consequence of calculating expectation of both sides of (6.1))

$$
1=A_{j k}^{(1)}+A_{j k}^{(2)}+\rho^{j+k} B_{j k}+C_{j k} .
$$

Secondly let us multiply (6.1) by $X_{n-j}^{2}, X_{n+k}^{2}$, and $X_{n-j} X_{n+k}$ and let us take expectation of both sides of obtained in that way equalities. In doing so we apply assertions of Lemma 3. In this way we will get three equations:

$$
\begin{aligned}
1+\rho^{2 j}(1+q)= & A_{j k}^{(1)}(2+q)+A_{j k}^{(2)}\left(1+\rho^{2(j+k)}(1+q)\right) \\
& +B_{j k} \rho^{j+k}(2+q)+1-A_{j k}^{(1)}-A_{j k}^{(2)}-\rho^{j+k} B_{j k}, \\
1+\rho^{2 k}(1+q)= & A_{j k}^{(1)}\left(1+\rho^{2(j+k)}(1+q)\right)+A_{j k}^{(2)}(2+q) \\
& +B_{j k} \rho^{j+k}(2+q)+1-A_{j k}^{(1)}-A_{j k}^{(2)}-\rho^{j+k} B_{j k} \\
\rho^{j+k}(2+q)= & A_{j k}^{(1)} \rho^{j+k}(2+q)+A_{j k}^{(2)} \rho^{j+k}(2+q) \\
& +B_{j k}\left(1+\rho^{2(j+k)}(1+q)\right) \\
& +\rho^{j+k}\left(1-A_{j k}^{(1)}-A_{j k}^{(2)}-\rho^{j+k} B_{j k}\right) .
\end{aligned}
$$

Solution of this system of equations is (3.4a), as it can be easily checked. Proposition 1 is proved.

Pro of of $\mathrm{Lemma} 2$. Consider discrete time random field $\mathbf{Z}=\left\{Z_{k}\right\}_{k \in \mathbf{Z}}$ such that $Z_{k}=X_{k j+m}$, for some fixed $j$ and $0 \leqslant m \leqslant j-1$. Obviously we have

$$
\begin{aligned}
\mathbf{E}\left(Z_{k} \mid \mathscr{F}_{\neq k}\right) & =\mathbf{E}\left(X_{k j+m} \mid \mathscr{F}_{(k-1) j+m, \geqslant(k+1) j+m}\right) \\
& =\frac{\rho^{j}}{1+\rho^{2 j}}\left(X_{(k-1) j+m}+X_{(k+1) j+m}\right)=\frac{\rho^{j}}{1+\rho^{2 j}}\left(Z_{k-1}+Z_{k+1}\right),
\end{aligned}
$$


and

$$
\begin{aligned}
\mathbf{E}\left(Z_{k}^{2} \mid \mathscr{F}_{\neq k}\right) & =\mathbf{E}\left(X_{k j+m}^{2} \mid \mathscr{F}_{k(k-1) j+m, \geqslant(k+1) j+m}\right) \\
& =A_{j}\left(X_{(k-1) j+m}^{2}+X_{(k+1) j+m}^{2}\right)+B_{j} X_{(k-1) j+m} X_{(k+1) j+m}+C_{j} \\
& =A_{j}\left(Z_{k-1}^{2}+Z_{k+1}^{2}\right)+B_{j} Z_{k-1} Z_{k+1}+C_{j},
\end{aligned}
$$

where $A_{j}=A_{j j}^{(1)}=A_{j j}^{(2)}, B_{j}=B_{j j}$. Thus $\mathbf{Z}$ is 1 TSP with different parameters. Notice that one-dimensional distributions of processes $\mathbf{X}$ and $\mathbf{Z}$ are the same. Hence parameters $q$ for both processes $\mathbf{Z}$ and $\mathbf{X}$ are the same. On the other hand, parameter $\rho_{Z}$ of the process $\mathbf{Z}$ is related to parameter $\rho$ of the process $\mathbf{X}$ by the following relationship:

$$
\rho_{Z}=\mathbf{E} Z_{0} Z_{1}=\mathbf{E} X_{0} X_{j}=\rho^{j} .
$$

Thus applying formulae (2.4) we get (3.4a).

$\mathrm{Pr}$ o of of $\mathrm{Th}$ e or e $\mathrm{m} 1$. From Lemma 2 of the previous section it follows that if for some $\delta>0, \mathbf{X}=\left\{X_{n}^{(\delta)}\right\}_{n \in \mathbf{Z}}$ is a regular 1TSP with some parameters $q$ and $\rho=\rho(\delta)$, then for every $j$ and $m \in\{0, \ldots, j-1\}$, process $Z_{k}^{(m)}=X_{k j+m}^{(\delta)}$ is also the 1TSP with the same parameter $q$ and parameter $\rho_{z}=\rho^{j}$. Notice however that $Z_{k}^{(0)}=X_{k}^{(j \delta)}$. Similarly if we considered process $\widehat{X}=\left\{\widehat{X}_{n}^{(\delta / j)}\right\}_{n \in \mathbf{Z}}$, then since $X_{n}^{(\delta)}=\widehat{X}_{n j}^{(\delta / j)}$ and one 1TSP is characterized by one parameter $q$ we deduce that processes $\mathbf{X}$ and $\widehat{X}$ share the same parameter $q$. Hence the fact that Condition $(\delta)$ is satisfied for every $\delta$ implies that all implied by it regular 1TSP are characterized by one, same parameter $q$. Further since for same $\delta>0$ regular 1TSP $\left\{X_{n}^{(\delta)}\right\}_{n \in \mathbf{Z}}$ is $L_{2}$-stationary with covariance function $K(n, m)=\rho(\delta)^{|n-m|}=\mathbf{E} X_{0}^{(\delta)} X_{|n-m|}^{(\delta)}$, we have also for any integer $k$ : $\rho(\delta)^{n}=\mathbf{E} X_{0}^{(\delta)} X_{n}^{(\delta)}=\mathbf{E} X_{0}^{(\delta / k)} X_{n k}^{(\delta / k)}=\rho(\delta / k)^{n k}$. Or equivalently we have

$$
\forall k \in \mathbf{Z}, \delta, \theta>0: \quad \rho(\delta)=\rho\left(\frac{\delta}{k}\right)^{k}, \quad \rho(k \theta)=\rho(\theta)^{k} .
$$

Now take $\delta=k \theta / m$ for some $\theta$. We will get then $\rho(k \theta / m)=\rho(\theta)^{k / m}$. Now let us take sequences of integers $\left\{k_{n}, m_{n}\right\}$ such that $k_{n} / m_{n} \rightarrow 1 / \theta, n \rightarrow \infty$, then, using $L_{2}$-continuity we get

$$
\rho(\theta)=\rho(1)^{\theta} .
$$

In other words, we deduce that if $\mathbf{Y}$ existed, then it would be $L_{2}$-stationary with covariance function

$$
K(t, s)=K(|s-t|)=\rho(1)^{|s-t|}
$$

for some $\rho(1) \in(0,1)$. Let us introduce new parameter

$$
\alpha=\ln \frac{1}{\rho(1)}>0
$$


We have then

$$
K(s, t)=\exp (-\alpha|s-t|) .
$$

Consequently the fact that Condition $(\delta)$ is satisfied for every $\delta$ implies that all implied by it regular 1TSP are characterized by one, same parameter $q$ and covariance function defined by same parameter $\alpha$.

Existence of $\mathbf{Y}$ will be shown for two cases separately. Since for $q=1$ we have normality of the one-dimensional and conditional distributions, we deduce that the process $\mathbf{Y}$ for $q=1$ is in fact the well-known OrnsteinUhlenbeck process.

Now let us consider fixed $q \in(-1,1)$. First we will deduce the existence of the process $\widetilde{\mathbf{Y}}=\left(Y_{t}\right)_{t \in \mathbf{Q}}$. This follows from Kolmogorov's extension theorem. Since having natural ordering of $\mathbf{Q}$ we need only consistency of the family of finite dimensional distributions of $\tilde{\mathbf{Y}}$. This can be however easily shown by the following argument. Let us take finite set of numbers $r_{1}<r_{2}<\cdots<r_{n}$ from $\mathbf{Q}$. Let $M$ denote the smallest common denominator of these numbers. Let us consider regular 1TSP $\mathbf{X}^{M}$ with $q$ and $\widehat{\alpha}=\alpha / M$. Note that then $X_{n}^{M}=Y_{n / M}$ for $n \in \mathbf{N}$. Then joint distribution of $\left(Y_{r_{1}}, \ldots, Y_{r_{n}}\right)$ is in fact a joint distribution of $\left(X_{R_{1}}^{M}, \ldots, X_{R_{n}}^{M}\right)$, where numbers $R_{1}, \ldots, R_{n}$ are defined by the relationships $r_{i}=R_{i} / M$. Since process $\mathbf{X}^{M}$ exists we have consistency because if $\left\{\tau_{1}, \ldots, \tau_{k}\right\} \subset\left\{r_{1}, \ldots, r_{n}\right\}$ then distribution of $\left(Y_{\tau_{1}}, \ldots, Y_{\tau_{k}}\right)$ being equal to the distribution of $\left(X_{T_{1}}^{M}, \ldots, X_{T_{k}}^{M}\right)$ with $T_{j}$ defined by $\tau_{j}=T_{j} / M$ for $j=1, \ldots, k$ is a projection of the distribution of $\left(Y_{r_{1}}, \ldots, Y_{r_{n}}\right)$. Hence we deduce that the process $\tilde{\mathbf{Y}}$ with values in the compact space $S(q)$ exists. Now we use separability theorem (see, e.g., $[24]$ ) and view $\tilde{\mathbf{Y}}$ as separable modification of the process $\mathbf{Y}$ itself. Hence the process $\mathbf{Y}$ exists.

P r o of of $\mathrm{Th}$ e or e m 3. i) The fact that $\mathbf{E} Y_{t}^{2}=1$ and that for $s \geqslant 0, \mathbf{D}\left(Y_{t+s} \mid Y_{t}\right)=1-e^{-2 \alpha s}$ follows from (4.3) and (4.4) and the definition of polynomials $p_{n}$ for $n=1,2$. If $q=1$, then we have OU process and the assertion is true. For $|q|<1$, we apply assertion of Theorem 3 in [24, Section 9.1] and the following estimation based on Chebyshev inequality:

$$
\begin{aligned}
\gamma_{\varepsilon}(t) & =\sup _{y \in S(q), t \leqslant h} \mathbf{P}\left(\left|Y_{s+t}-Y_{s}\right| \geqslant \varepsilon \mid Y_{s}=y\right) \\
& =\frac{\mathbf{E}\left|Y_{t+s}-Y_{s}\right|^{2}}{\varepsilon^{2}} \leqslant \frac{2 \mathbf{D}\left(Y_{t+s} \mid Y_{s}=y\right)+2 \mathbf{E}\left(\left|Y_{s}-e^{-\alpha t} Y_{s}\right|^{2} \mid Y_{s}=y\right)}{\varepsilon^{2}} \\
& =\frac{2\left(1-e^{-2 \alpha t}\right)+2 y^{2}\left(1-e^{-\alpha t}\right)^{2}}{\varepsilon^{2}} \cong \frac{4 \alpha t+8 \alpha^{2} t^{2} /(1-q)}{\varepsilon^{2}}
\end{aligned}
$$

from which it follows that for all $\varepsilon>0, \gamma_{\varepsilon}(t) \rightarrow 0$ as $t \rightarrow 0$. Another justification of this assertion follows properties of martingales and is given below.

ii) Following observations: 1. $q$-Hermite polynomials are the orthogonal basis of the space denoted by $B(q)$. 2. Conditional distributions of $Y_{t+s} \mid Y_{t}=$ 
$y$ having densities $f_{C N}\left(x \mid y, e^{-\alpha s}, q\right)$ form a continuous semigroup following Lemma 1, iv). 3. (4.6) follows Lemma 1, ii).

iii) If $q=1$, then we deal with classical OU process that both has Feller property and is strongly Markov. For $|q|<1$ firstly we use the fact that $\left|H_{i}(x \mid q)\right| \leqslant W_{i}(q) /(1-q)^{i / 2}$ by Lemma $\left.1, \mathrm{v}\right)$, secondly we use (4.6) together with Lemma 1 , vi) which guarantees that

$$
\max _{x \in S(q)}\left|P^{\tau}(f)(x)\right| \leqslant \frac{\max _{j \geqslant 0}\left|b_{j} \sqrt{(q)_{j}}\right|}{(\exp (-\alpha \tau))_{\infty}^{2}}
$$

where coefficients $b_{i}$ are defined by

$$
B(q) \ni f(x)=\sum_{j \geqslant 0} \frac{b_{j}}{\sqrt{[j]_{q} !}} H_{j}(x \mid q) .
$$

Thus if $f$ was continuous and bounded, then $P^{\tau}(f)(x)$ is also continuous and bounded. Hence we have Feller property. To get strong Markov property we use Theorem 1 of [24, Section 9.2] that asserts that every time homogeneous Markov family, having cádlág trajectories and Feller property, is also strongly Markov.

iv) Consider function $f \in B(q)$ and take $n_{0} \in \mathbf{N}$ such that

$$
\max \left(\sup _{x \in S(q)}\left|\sum_{n \geqslant n_{0}} \frac{b_{n}}{\sqrt{[n]_{q} !}} H_{n}(x \mid q)\right|, \sup _{x \in S(q)}\left|\sum_{n \geqslant n_{0}} e^{-n \alpha \tau} \frac{b_{n}}{\sqrt{[n]_{q} !}} H_{n}(x \mid q)\right|\right) \leqslant \varepsilon
$$

for some chosen beforehand $\varepsilon>0$. Since on the compact space $S(q)$ uniform convergence to a continuous function is equivalent to pointwise convergence and since from $L_{2}$-convergence follows existence of a subsequence $\left\{k_{n}\right\}$ such that $\sum_{j \geqslant 0}^{k_{n}}\left(b_{j} / \sqrt{[j]_{q} !}\right) H_{j}(x \mid q)$ converges pointwise to its continuous limit, we deduce that such $n_{0}$ exists. Now we notice that

$$
\sup _{x \in S(q)}\left|f(x)-P^{\tau}(f)(x)\right| \leqslant 2 \varepsilon+\sup _{x \in S(q)}\left|\sum_{j=0}^{n_{0}-1}\left(1-e^{-j \alpha \tau}\right) \frac{b_{j}}{\sqrt{[j]_{q} !}} H_{j}(x \mid q)\right| \leqslant 3 \varepsilon,
$$

if only $\tau$ is sufficiently small. Hence

$$
\lim _{\tau \rightarrow 0^{+}} \sup _{x \in S(q)}\left|f(x)-P^{\tau}(f)(x)\right|=0,
$$

and consequently we see that $\left\{P^{\tau}(f)\right\}_{\tau \geqslant 0}$ is right-continuous meaning that process $\mathbf{Y}$ is a Feller process. Thus the infinitesimal operator $A$ exists. Its value on $H_{n}(x \mid q)$ can be found by the following argument:

$$
\begin{aligned}
A H_{n}(x \mid q) & =\lim _{h \downarrow 0^{+}} \frac{1}{h}\left[\mathbf{E}\left(H_{n}\left(Y_{t+h} \mid q\right) \mid Y_{t}=x\right)-H_{n}(x \mid q)\right] \\
& =\lim _{h \downarrow 0^{+}} \frac{1}{h}\left(e^{-\alpha n h} H_{n}(x \mid q)-H_{n}(x \mid q)\right)=-n \alpha H_{n}(x \mid q) .
\end{aligned}
$$


Theorem 3 is proved.

Pr o of of $\mathrm{Th}$ e or e $\mathrm{m}$ 5. 1. Suppose $\tau<\sigma$, then we have

$$
\begin{aligned}
\operatorname{cov}\left(X_{\tau}, X_{\sigma}\right) & =\sqrt{\tau \sigma} \mathbf{E} Y_{(\log \tau) /(2 \alpha)} Y_{(\log \sigma) /(2 \alpha)}=\sqrt{\tau \sigma} e^{-\alpha|(\log \tau) /(2 \alpha)-(\log \sigma) /(2 \alpha)|} \\
& =\sqrt{\tau \sigma} e^{-\log (\sigma / \tau) / 2}=\sqrt{\tau \sigma} \sqrt{\frac{\sigma}{\tau}}=\sigma
\end{aligned}
$$

2) \& 3) We have $\mathbf{P}\left(X_{t} \leqslant x\right)=\mathbf{P}\left(\sqrt{\tau} Y_{(\log \tau) /(2 \alpha)} \leqslant x\right)=\mathbf{P}\left(Y_{(\log \tau) /(2 \alpha)} \leqslant\right.$ $x / \sqrt{\tau})$. Knowing that $f_{N}$ is a density of $Y_{t}$ we get immediately assertion 2 . To get assertion 3 we calculate:

$$
\begin{aligned}
\mathbf{P}\left(X_{\tau}-X_{\sigma} \leqslant x \mid X_{\sigma}=y\right) & =\mathbf{P}\left(X_{\tau} \leqslant x+y \mid X_{\sigma}=y\right) \\
& =\mathbf{P}\left(\sqrt{\tau} Y_{(\log \tau) /(2 \alpha)} \leqslant x+y \mid \sqrt{\sigma} Y_{(\log \sigma) /(2 \alpha)}=y\right) \\
& =\mathbf{P}\left(Y_{(\log \tau) /(2 \alpha)} \leqslant \frac{x+y}{\sqrt{\tau}} \mid Y_{(\log \sigma) /(2 \alpha)}=\frac{y}{\sqrt{\sigma}}\right) .
\end{aligned}
$$

Now we recall that $f_{C N}\left(x \mid y, q, e^{-\alpha|s-t|}\right)$ is the density of $Y_{t}$ given $Y_{s}=y$, by Theorem 2.

4) Notice that $X_{\tau} / \sqrt{\tau}=Y_{(\log \tau) /(2 \alpha)}$. We have using assertion 5) of Theorem 2:

$$
\begin{aligned}
& \mathbf{E}\left(\tau^{n / 2} H_{n}\left(\frac{X_{\tau}}{\sqrt{\tau}}\right) \mid \mathscr{F}_{\leqslant \sigma}^{X}\right)=\tau^{n / 2} \mathbf{E}\left(H_{n}\left(Y_{(\log \tau) /(2 \alpha)}\right) \mid \mathscr{F}_{(\log \sigma) /(2 \alpha)}\right) \\
& \quad=\tau^{n / 2} e^{-\alpha n((\log \tau) /(2 \alpha)-(\log \sigma) /(2 \alpha))} H_{n}\left(Y_{(\log \sigma) /(2 \alpha)}\right)=\sigma^{n / 2} H_{n}\left(\frac{X_{\sigma}}{\sqrt{\sigma}}\right) \quad \text { a.s. }
\end{aligned}
$$

Similarly

$$
\begin{gathered}
\mathbf{E}\left(\sigma^{-n / 2} H_{n}\left(\frac{X_{\sigma}}{\sqrt{\sigma}} \mid q\right) \mid \mathscr{F}_{\geqslant \tau}^{X}\right)=\sigma^{-n / 2} \mathbf{E}\left(H_{n}\left(Y_{(\log \sigma) /(2 \alpha)} \mid q\right) \mid \mathscr{F}_{\geqslant(\log \tau) /(2 \alpha)}\right) \\
=\sigma^{-n / 2} e^{-\alpha((\log \tau) /(2 \alpha)-(\log \sigma) /(2 \alpha))} H_{n}\left(Y_{(\log \tau) /(2 \alpha)} \mid q\right)=\tau^{-n / 2} H_{n}\left(\frac{X_{\tau}}{\sqrt{\tau}} \mid q\right) .
\end{gathered}
$$

5) To get the first part we proceed as follows:

$$
\begin{aligned}
& \sqrt{\sigma} \mathbf{E}\left(Y_{(\log \sigma) /(2 \alpha)} \mid \mathscr{F}_{\leqslant \log (\sigma-\delta) /(2 \alpha), \geqslant \log (\sigma+\gamma) /(2 \alpha)}\right) \\
& =\sqrt{\sigma}\left(\frac{\sqrt{(\sigma-\delta) / \sigma}(1-\sigma /(\sigma+\gamma))}{1-(\sigma-\delta) /(\sigma+\gamma)} Y_{\log (\sigma-\delta) /(2 \alpha)}\right. \\
& \left.+\frac{\sqrt{\sigma /(\sigma+\gamma)}(1-(\sigma-\delta) / \sigma)}{1-(\sigma-\delta) /(\sigma+\gamma)} Y_{\log (\sigma+\gamma) /(2 \alpha)}\right) \\
& =\frac{\gamma}{\delta+\gamma} X_{\sigma-\delta}+\frac{\delta}{\delta+\gamma} X_{\sigma+\gamma} \text {. }
\end{aligned}
$$


To get the second part we calculate:

$$
\begin{aligned}
& \mathbf{E}\left(X_{\sigma}^{2} \mid \mathscr{F}_{\leqslant \sigma-\delta, \geqslant \sigma+\gamma}^{X}\right)=\sigma \mathbf{E}\left(Y_{(\log \sigma) /(2 \alpha)}^{2} \mid \mathscr{F}_{\leqslant \log (\sigma-\delta) /(2 \alpha), \geqslant \log (\sigma+\gamma) /(2 \alpha)}\right) \\
& =\sigma \frac{\frac{\sigma-\delta}{\sigma}\left(1-\frac{\sigma}{\sigma+\gamma}\right)\left(1-\frac{q \sigma}{\sigma+\gamma}\right)}{\left(1-\frac{\sigma-\delta}{\sigma+\gamma}\right)\left(1-q \frac{\sigma-\delta}{\sigma+\gamma}\right)} Y_{\log (\sigma-\delta) /(2 \alpha)}^{2} \\
& +\sigma \frac{\frac{\sigma}{\sigma+\gamma}\left(1-\frac{\sigma-\delta}{\sigma}\right)\left(1-q \frac{\sigma-\delta}{\sigma}\right)}{\left(1-\frac{\sigma-\delta}{\sigma+\gamma}\right)\left(1-q \frac{\sigma-\delta}{\sigma+\gamma}\right)} Y_{\log (\sigma+\gamma) /(2 \alpha)}^{2} \\
& +\sigma \frac{(q+1) \sqrt{\frac{\sigma-\delta}{\sigma+\gamma}}\left(1-\frac{\sigma}{\sigma+\gamma}\right)\left(1-\frac{\sigma-\delta}{\sigma}\right)}{\left(1-\frac{\sigma-\delta}{\sigma+\gamma}\right)\left(1-q \frac{\sigma-\delta}{\sigma+\gamma}\right)} Y_{\log (\sigma-\delta) /(2 \alpha)} Y_{\log (\sigma+\gamma) /(2 \alpha)} \\
& +\sigma \frac{\left(1-\frac{\sigma-\delta}{\sigma}\right)\left(1-\frac{\sigma}{\sigma+\gamma}\right)}{\left(1-q \frac{\sigma-\delta}{\sigma+\gamma}\right)} \text {. }
\end{aligned}
$$

Keeping in mind that $X_{\tau}=\sqrt{\tau} Y_{(\log \tau) /(2 \alpha)}$ we get:

$$
\begin{aligned}
\mathbf{E}\left(X_{\sigma}^{2} \mid \mathscr{F}_{\leqslant \sigma-\delta, \geqslant \sigma+\gamma}^{X}\right) \\
=\frac{\gamma((1-q) \sigma+\gamma)}{(\delta+\gamma)(\sigma(1-q)+\gamma+q \delta)} X_{\sigma-\delta}^{2}+\frac{\delta((1-q) \sigma+q \delta)}{(\delta+\gamma)(\sigma(1-q)+\gamma+q \delta)} X_{\sigma+\gamma}^{2} \\
\quad+\frac{(q+1) \delta \gamma}{(\delta+\gamma)(\sigma(1-q)+\gamma+q \delta)} X_{\sigma-\delta} X_{\sigma+\gamma}+\frac{\delta \gamma}{\sigma(1-q)+\gamma+q \delta} .
\end{aligned}
$$

Theorem 5 is proved.

Pro of of $\mathrm{C}$ or ollary 2. To prove i) we calculate:

$$
\begin{aligned}
& \mathbf{E}\left(\left(X_{\tau}-X_{\sigma}\right)^{2} \mid \mathscr{F}_{\leqslant \sigma}^{X}\right) \\
& =\mathbf{E}\left(X_{\tau}^{2} \mid \mathscr{F}_{\leqslant \sigma}^{X}\right)-2 X_{\sigma}^{2}+X_{\sigma}^{2}=X_{\sigma}^{2}+\tau-\sigma-X_{\sigma}^{2}=\tau-\sigma, \\
& \mathbf{E}\left(\left(X_{\tau}-X_{\sigma}\right)^{3} \mid \mathscr{F}_{\leqslant \sigma}^{X}\right) \\
& =\mathbf{E}\left(X_{\tau}^{3} \mid \mathscr{F}_{\leqslant \sigma}^{X}\right)-3 X_{\sigma} \mathbf{E}\left(X_{\tau}^{2} \mid \mathscr{F}_{\leqslant \sigma}^{X}\right)+3 X_{\sigma}^{2} \mathbf{E}\left(X_{\tau} \mid \mathscr{F}_{\leqslant \sigma}^{X}\right)-X_{\sigma}^{3} \\
& =\mathbf{E}\left(X_{\tau}^{3} \mid \mathscr{F}_{\leqslant \sigma}^{X}\right)-3 X_{\sigma} \mathbf{E}\left(X_{\tau}^{2} \mid \mathscr{F}_{\leqslant \sigma}^{X}\right)+2 X_{\sigma}^{3}, \\
& \mathbf{E}\left(\left(X_{\tau}-X_{\sigma}\right)^{4} \mid \mathscr{F}_{\leqslant \sigma}^{X}\right)=\mathbf{E}\left(X_{\tau}^{4} \mid \mathscr{F}_{\leqslant \sigma}^{X}\right)-4 X_{\sigma} \mathbf{E}\left(X_{\tau}^{3} \mid \mathscr{F}_{\leqslant \sigma}^{X}\right) \\
& \quad+6 X_{\sigma}^{2} \mathbf{E}\left(X_{\tau}^{2} \mid \mathscr{F}_{\leqslant \sigma}^{X}\right)-4 X_{\sigma}^{3} \mathbf{E}\left(X_{\tau} \mid \mathscr{F}_{\leqslant \sigma}^{X}\right)+X_{\sigma}^{4} \\
& =\mathbf{E}\left(X_{\tau}^{4} \mid \mathscr{F}_{\leqslant \sigma}^{X}\right)-4 X_{\sigma} \mathbf{E}\left(X_{\tau}^{3} \mid \mathscr{F}_{\leqslant \sigma}^{X}\right)+3 X_{\sigma}^{4}+6(\tau-\sigma) X_{\sigma}^{2} .
\end{aligned}
$$

Now recalling that $H_{2}(x \mid q)=x^{2}-1, H_{3}(x \mid q)=x^{3}-(2+q) x$, and $H_{4}(x \mid q)=x^{4}-\left(3+2 q+q^{2}\right) x^{2}+\left(1+q+q^{2}\right)$ we see that:

$$
\begin{aligned}
\sigma H_{2}\left(\frac{X_{\sigma}}{\sqrt{\sigma}} \mid q\right) & =\mathbf{E}\left(\tau H_{2}\left(\frac{X_{\tau}}{\sqrt{\tau}} \mid q\right) \mid \mathscr{F}_{\leqslant \sigma}^{X}\right), \\
\sigma^{3 / 2} H_{3}\left(\frac{X_{\sigma}}{\sqrt{\sigma}} \mid q\right) & =\mathbf{E}\left(\tau^{3 / 2} H_{3}\left(\frac{X_{\tau}}{\sqrt{\tau}} \mid q\right) \mid \mathscr{F}_{\leqslant \sigma}^{X}\right)
\end{aligned}
$$




$$
\begin{aligned}
= & \mathbf{E}\left(X_{\tau}^{3} \mid \mathscr{F}_{\leqslant \sigma}^{X}\right)-\tau(2+q) \mathbf{E}\left(X_{\tau} \mid \mathscr{F}_{\leqslant \sigma}^{X}\right), \\
\sigma^{2} H_{4}\left(\frac{X_{\sigma}}{\sqrt{\sigma}} \mid q\right)= & \mathbf{E}\left(\tau^{2} H_{4}\left(\frac{X_{t}}{\sqrt{\tau}} \mid q\right) \mid \mathscr{F}_{\leqslant \sigma}^{X}\right) \\
= & \mathbf{E}\left(X_{\tau}^{4} \mid \mathscr{F}_{\leqslant \sigma}^{X}\right)-\tau\left(3+2 q+q^{2}\right) \mathbf{E}\left(X_{\tau}^{2} \mid \mathscr{F}_{\leqslant \sigma}^{X}\right) \\
& +\left(1+q+q^{2}\right) \tau^{2} .
\end{aligned}
$$

Thus

$\mathbf{E}\left(X_{\tau}^{2} \mid \mathscr{F}_{\leqslant \sigma}^{X}\right)=X_{\sigma}^{2}+\tau-\sigma$,

$\mathbf{E}\left(X_{\tau}^{3} \mid \mathscr{F}_{\leqslant \sigma}^{X}\right)=\tau(2+q) X_{\sigma}+X_{\sigma}^{3}-\sigma(2+q) X_{\sigma}=(\tau-\sigma)(2+q) X_{\sigma}+X_{\sigma}^{3}$,

$\mathbf{E}\left(X_{\tau}^{4} \mid \mathscr{F}_{\leqslant \sigma}^{X}\right)=X_{\sigma}^{4}+(\tau-\sigma)\left(3+2 q+q^{2}\right) X_{\sigma}^{2}-(\tau-\sigma)(\tau+\sigma)\left(1+q+q^{2}\right)$.

So

$$
\begin{aligned}
\mathbf{E}\left(\left(X_{\tau}-X_{\sigma}\right)^{3} \mid \mathscr{F}_{\leqslant \sigma}^{X}\right)= & (\tau-\sigma)(2+q) X_{\sigma}+X_{\sigma}^{3}-3 X_{\sigma}\left(X_{\sigma}^{2}+\tau-\sigma\right)+2 X_{\sigma}^{3} \\
= & -(1-q)(\tau-\sigma) X_{\sigma}, \mathbf{E}\left(\left(X_{\tau}-X_{\sigma}\right)^{4} \mid \mathscr{F}_{\leqslant \sigma}^{X}\right) \\
= & 3 X_{\sigma}^{4}+6(\tau-\sigma) X_{\sigma}^{2}+X_{\sigma}^{4}+(\tau-\sigma)\left(3+2 q+q^{2}\right) X_{\sigma}^{2} \\
& +(\tau-\sigma) \tau\left(3+2 q+q^{2}\right)-\left(1+q+q^{2}\right)\left(\tau^{2}-\sigma^{2}\right) \\
& -4 X_{\sigma}^{4}-4(\tau-\sigma)(2+q) X_{\sigma}^{2} \\
= & X_{\sigma}^{2}(\tau-\sigma)(1-q)^{2}+(2+q)(\tau-\sigma)^{2} \\
& +(\tau-\sigma) \sigma\left(1-q^{2}\right) .
\end{aligned}
$$

If $\mathbf{X}$ had independent increments, then $\mathbf{E}\left(\left(X_{\tau}-X_{\sigma}\right)^{4} \mid \mathscr{F}_{\leqslant \sigma}^{X}\right)$ would not depend on $X_{\sigma}^{2}$. Note that for $q=1$ we have $\mathbf{E}\left(\left(X_{\tau}-X_{\sigma}\right)^{4} \mid \mathscr{F}_{\leqslant \sigma}^{X}\right)=$ $3(\tau-\sigma)^{2}=3 \mathbf{E}\left(\left(X_{\tau}-X_{\sigma}\right)^{2} \mid \mathscr{F}_{\leqslant \sigma}^{X}\right)$.

ii) Follows properties of martingales. Thus almost every path of the process $\mathbf{X}$ has no oscillatory discontinuities, in other words has at every point $t$ left- and right-hand side limit. Since process $\mathbf{Y}$ is obtained from the process $\mathbf{X}$ by continuous transformation it has similar properties.

iii) Follows the fact that the $q$-Wiener process is obtained from the $(\alpha, q)$-OU process by continuous (even smooth) transformation (4.9). On its side the $(\alpha, q)$-OU has the Feller property and is strongly Markov as shown in Theorem 3, iii).

Corollary 2 is proved.

$\mathrm{Pr}$ o of of $\mathrm{C}$ or o $1 \mathrm{lary} 3$. Following given formulae we have for $n=1$ and $n=2$ :

$$
\begin{aligned}
A_{0,0}^{(1)} & =\frac{\gamma}{\gamma+\delta} \sqrt{\frac{\sigma-\delta}{\sigma}}, \quad A_{0,1}^{(1)}=\frac{\delta}{\gamma+\delta} \sqrt{\frac{\sigma+\gamma}{\sigma}} \\
A_{0,-1}^{(2)} & =\frac{\gamma \delta}{\sigma(\gamma+\delta)(\gamma+q \delta+(1-q) \sigma)} \frac{(\sigma-\delta)(\gamma+\sigma(1-q))}{\delta},
\end{aligned}
$$




$$
\begin{aligned}
A_{0,0}^{(2)} & =\frac{\gamma \delta}{\sigma(\gamma+\delta)(\gamma+q \delta+(1-q) \sigma)}[2]_{q} \sqrt{(\sigma-\delta)(\sigma+\gamma)} \\
A_{0,1}^{(2)} & =\frac{\gamma \delta}{\sigma(\gamma+\delta)(\gamma+q \delta+(1-q) \sigma)} \frac{(\sigma+\gamma)(q \delta+(1-q) \sigma)}{\gamma} \\
A_{1,0}^{(2)} & =-[2]_{q} \frac{\gamma \delta(\sigma-\delta)}{\sigma(\gamma+\delta)(\gamma+q \delta+(1-q) \sigma)}
\end{aligned}
$$

Now we get immediately (4.15a) and (4.15b). To get (4.16) we perform the following calculations:

$$
\mathbf{D}\left(X_{\sigma} \mid \mathscr{F}_{\leqslant \sigma-\delta, \geqslant \sigma+\gamma}^{X}\right)=\mathbf{E}\left(X_{\sigma}^{2} \mid \mathscr{F}_{\leqslant \sigma-\delta, \geqslant \sigma+\gamma}^{X}\right)-\left(\mathbf{E}\left(X_{\sigma} \mid \mathscr{F}_{\leqslant \sigma-\delta, \geqslant \sigma+\gamma}^{X}\right)\right)^{2} .
$$

Hence

$$
\begin{aligned}
& \mathbf{D}\left(X_{\sigma} \mid \mathscr{F}_{\leqslant \sigma-\delta, \geqslant \sigma+\gamma}^{X}\right) \\
&= \frac{\delta \gamma}{\sigma(1-q)+\gamma+q \delta}+X_{\sigma-\delta}^{2}\left(\frac{\gamma((1-q) \sigma+\gamma)}{(\delta+\gamma)(\sigma(1-q)+\gamma+q \delta)}-\frac{\gamma^{2}}{(\delta+\gamma)^{2}}\right) \\
&+X_{\sigma+\gamma}^{2}\left(\frac{\delta((1-q) \sigma+q \delta)}{(\delta+\gamma)(\sigma(1-q)+\gamma+q \delta)}-\left(\frac{\delta}{\delta+\gamma}\right)^{2}\right) \\
&+X_{\sigma-\delta} X_{\sigma+\gamma}\left(\frac{(q+1) \delta \gamma}{(\delta+\gamma)(\sigma(1-q)+\gamma+q \delta)}-\frac{2 \gamma \delta}{(\delta+\gamma)^{2}}\right) .
\end{aligned}
$$

After some simplifications we get

$$
\begin{aligned}
\mathbf{D}( & \left.X_{\sigma} \mid \mathscr{F}_{\leqslant \sigma-\delta, \geqslant \sigma+\gamma}^{X}\right) \\
= & \frac{\delta \gamma}{\sigma(1-q)+\gamma+q \delta}+\frac{(1-q) \delta \gamma(\sigma+\gamma)}{(\gamma+\delta)^{2}(\sigma(1-q)+\gamma+q \delta)} X_{\sigma-\delta}^{2} \\
& +\frac{(1-q) \delta \gamma(\sigma-\delta)}{(\gamma+\delta)^{2}(\sigma(1-q)+\gamma+q \delta)} X_{\sigma+\gamma}^{2} \\
& -\frac{(1-q) \gamma \delta(2 \sigma-\delta+\gamma)}{(\gamma+\delta)^{2}(\sigma(1-q)+\gamma+q \delta)} X_{\sigma-\delta} X_{\sigma+\gamma} \\
= & \frac{\delta \gamma}{(\sigma(1-q)+\gamma+q \delta)(\gamma+\delta)^{2}} \\
& \times\left((\gamma+\delta)^{2}+(1-q)\left(X_{\sigma+\gamma}-X_{\sigma-\delta}\right)\left((\sigma-\delta) X_{\sigma+\gamma}-(\sigma+\gamma) X_{\sigma-\delta}\right)\right) .
\end{aligned}
$$

Corollary 3 is proved.

P r o of of $\mathrm{Re} \mathrm{mark} 14$. We have:

$$
\begin{aligned}
\operatorname{cov}\left(\left(X_{\tau}-X_{\sigma}\right)^{2},\left(X_{\omega}-X_{v}\right)^{2}\right)= & \mathbf{E}\left(\left(X_{\tau}-X_{\sigma}\right)^{2} \mathbf{E}\left(\left(X_{\omega}-X_{v}\right)^{2} \mid \mathscr{F}_{\leqslant \tau}^{X}\right)\right) \\
& -(\omega-v)(\tau-\sigma)=0, \\
\operatorname{cov}\left(\left(X_{\tau}-X_{\sigma}\right)^{3},\left(X_{\omega}-X_{v}\right)^{3}\right)= & -(1-q)(\omega-v) \mathbf{E} X_{v}\left(X_{\tau}-X_{\sigma}\right)^{3} \\
= & -(1-q)(\omega-v) \mathbf{E} X_{\tau}\left(X_{\tau}-X_{\sigma}\right)^{3}
\end{aligned}
$$




$$
\begin{aligned}
= & -(1-q)(\omega-v) \mathbf{E} X_{\sigma}\left(X_{\tau}-X_{\sigma}\right)^{3}, \\
-(1-q)(\omega-v) \mathbf{E}\left(X_{\tau}-X_{\sigma}\right)^{4}= & -(1-q)(\tau-\sigma)(\omega-v) \\
& \times(\tau(2+q)-\sigma(1+2 q)) .
\end{aligned}
$$

Remark 14 is proved.

Pro of of Corollary 4. We multiply both sides of both formulae given in assertion 4) of Theorem 5 by $s^{n} /[n]_{q}$ and sum over $n$ from 0 to $\infty$. Now one has to use the fact that such a sum is absolutely convergent in the case of (4.10) for $s^{2} \sigma(1-q)<1$, while in the case of (4.11) for $s^{2}(1-q) / \sigma<1$. Changing order of summation and conditional expectation and applying formula 1.4 of [3] giving generating function of the $q$-Hermite polynomials, leads to (4.17) and (4.18), respectively. For $q=1$ we use the same formulae but this time multiplied by $s^{n} / n$ ! and after summing up with respect to $n$ we apply well-known formula for generating functions of Hermite polynomials. Corollary 4 is proved.

Acknowledgment. The author is grateful to an unknown referee for pointing out subtle questions concerning some proofs, hasty thoughtlessness in posing open questions and numerous misprints.

\section{REFERENCES}

1. Bryc W. Stationary random fields with linear regressions. - Ann. Probab., 2001, v. 29 , № 1 , p. 504-519.

2. Bryc W. Stationary Markov chains with linear regressions. - Stochastic Process. Appl., 2001, v. 93, № 2, p. 339-348.

3. Bryc W., Matysiak W., Szabtowski P. J. Probabilistic aspects of Al-Salam-Chihara polynomials. - Proc. Amer. Math. Soc., 2005, v. 133, № 4, p. 1127-1134.

4. Bressoud D. M. A simple proof of Mehler's formula for $q$-Hermite polynomials. Indiana Univ. Math. J., 1980, v. 29, № 4, p. 577-580.

5. Ismail M. E. H. Classical and Quantum Orthogonal Polynomials in One Variable. Cambridge: Cambridge University Press, 2005, 706 p.

6. Andrews G.E., Askey R., Roy R. Special Functions. Cambridge: Cambridge University Press, 1999, 664 p.

7. Matysiak W., Szabtowski P. J. A few remarks on Bryc's paper on random fields with linear regressions. - Ann. Probab., 2002, v. 30, № 3, p. 1486-1491.

8. Matysiak W., Szabtowski P. J. Bryc's random fields: the existence and distributions analysis, arXiv:math/0507296, 2005.

9. Bryc W., Wesotowski J. Conditional moments of $q$-Meixner processes. - Probab. Theory Related Fields, 2005, v. 131, № 3, p. 415-441.

10. Bryc W., Wesotowski J. Bi-Poisson process. - Infin. Dimens. Anal. Quantum Probab. Relat. Top., 2007, v. 10, № 2, p. 277-291.

11. Bożejko M., Bryc W. On a class of free Lévy laws related to a regression problem. J. Funct. Anal., 2006, v. 236, № 1, p. 59-77.

12. Anshelevich M., Belinschi S. T., Bożejko M., Lehner F. Free infinite divisibility for q-Gaussians. - Math. Res. Lett., 2010, v. 17, № 5, p. 905-916.

13. Bryc W., Matysiak W., Wesotowski J. The bi-Poisson process: a quadratic harness. Ann. Probab., 2008, v. 36, № 2, p. 623-646. 
14. Szabtowski P. J. Probabilistic implications of symmetries of $q$-Hermite and Al-SalamChihara polynomials. - Infin. Dimens. Anal. Quantum Probab. Relat. Top., 2008, v. 11, № 4, p. 513-522.

15. Szabtowski P.J. Multidimensional $q$-normal and related distributions - Markov case. - Electron. J. Probab., 2010, v. 15, № 40, p. 1296-1318.

16. Szabtowski P.J. On the structure and probabilistic interpretation of Askey-Wilson densities and polynomials with complex parameters. - J. Funct. Anal., 2011, v. 261, № 3, p. 635-659. http://arxiv.org/abs/1011.1541.

17. Szabtowski P.J. Expansions of one density via polynomials orthogonal with respect to the other. - J. Math. Anal. Appl., 2011, v. 383, №1, p. 35-54, http://arxiv.org/abs/1011.1492.

18. Szabtowski P.J. q-Gaussian distributions: simplifications and simulations. - J. Probab. Statist., 2009, Art. ID 752430, 18 p.

19. Wesotowski J. A martingale characterization of the Wiener process. - Statist. Probab. Lett., 1990, v. 10, № 3, p. 213-215.

20. Bożejko M., Kümmerer B., Speicher R. q-Gaussian processes: non-commutative and classical aspects. - Comm. Math. Phys., 1997, v. 185, № 1, p. 129-154.

21. Anshelevich M. $q$-Lévy processes. - J. Reine Angew. Math., 2004, v. 576, p. 181-207.

22. Anshelevich $M$. Generators of some non-commutative stochastic processes. arXiv:1104.1381, 2011.

23. Bryc $W$. Classical versions of $q$-Gaussian processes: conditional moments and Bell's inequality. - Comm. Math. Phys., 2001, v. 219, № 2, p. 259-270.

24. Вентцель А. Д. Курс теории случайных процессов. М.: Наука, 1975, 319 с.

Поступила в редакцию

03.VIII. 2010

Исправленный вариант 24.VII. 2011 